Algebraic 85 Geometric Topology

Volume 3 (2003) 235-285

Published: 5 March 2003

ATG

\title{
Heegaard diagrams and surgery descriptions for twisted face-pairing 3-manifolds
}

\author{
J.W. CANNON \\ W.J. FLOYD \\ W.R. PARRY
}

\begin{abstract}
The twisted face-pairing construction of our earlier papers gives an efficient way of generating, mechanically and with little effort, myriads of relatively simple face-pairing descriptions of interesting closed 3-manifolds. The corresponding description in terms of surgery, or Dehn-filling, reveals the twist construction as a carefully organized surgery on a link.

In this paper, we work out the relationship between the twisted face-pairing description of closed 3-manifolds and the more common descriptions by surgery and Heegaard diagrams. We show that all Heegaard diagrams have a natural decomposition into subdiagrams called Heegaard cylinders, each of which has a natural shape given by the ratio of two positive integers. We characterize the Heegaard diagrams arising naturally from a twisted face-pairing description as those whose Heegaard cylinders all have integral shape. This characterization allows us to use the Kirby calculus and standard tools of Heegaard theory to attack the problem of finding which closed, orientable 3-manifolds have a twisted face-pairing description.
\end{abstract}

AMS Classification $57 \mathrm{~N} 10$

Keywords 3-manifold constructions, Dehn surgery, Heegaard diagrams

\section{Introduction}

The twisted face-pairing construction of our earlier papers [1], [2], [3] gives an efficient way of generating, mechanically and with little effort, myriads of relatively simple face-pairing descriptions of interesting closed 3-manifolds. Starting with a faceted 3-ball $P$ and an arbitrary orientation-reversing face-pairing $\epsilon$ on $P$, one constructs a faceted 3-ball $Q$ and an orientation-reversing face-pairing $\delta$ on $Q$ such that the quotient $Q / \delta$ is a manifold. Here $Q$ is obtained from $P$ by subdividing the edges according to a function which assigns a positive integer (called a multiplier) to each edge cycle, and $\delta$ is obtained from $\epsilon$ by 
precomposing each face-pairing map with a twist. Which direction to twist depends on the choice of an orientation of $P$. Hence for a given faceted 3-ball $P$, orientation-reversing face-pairing $\epsilon$, and multiplier function, one obtains two twisted face-pairing manifolds $M=Q / \delta$ and $M^{*}=Q / \delta^{*}$ (one for each orientation of $P$ ).

In [1] and [2] we introduced twisted face-pairing 3-manifolds and developed their first properties. A surprising result in [2] is the duality theorem that says that, if $P$ is a regular faceted 3 -ball, then $M$ and $M^{*}$ are homeomorphic in a way that makes their cell structures dual to each other. This duality is instrumental in [3], where we investigated a special subset of these manifolds, the ample twisted face-pairing manifolds. We showed that the fundamental group of every ample twisted face-pairing manifold is Gromov hyperbolic with space at infinity a 2 -sphere.

In this paper we connect the twisted face-pairing construction with two standard 3 -manifold constructions. Starting with a faceted 3-ball $P$ with $2 g$ faces and an orientation-reversing face-pairing $\epsilon$ on $P$, we construct a closed surface $S$ of genus $g$ and two families $\gamma$ and $\beta$ of pairwise disjoint simple closed curves on $S$. The elements of $\gamma$ correspond to the face pairs and the elements of $\beta$ correspond to the edge cycles of $\epsilon$. Given a choice of multipliers for the edge cycles, we then give a Heegaard diagram for the resulting twisted face-pairing 3 -manifold. The surface $S$ is the Heegaard surface, and the family $\gamma$ is one of the two families of meridian curves. The other family is obtained from $\gamma$ by a product of powers of Dehn twists along elements of $\beta$; the powers of the Dehn twists are the multipliers. From the Heegaard diagram, one can easily construct a framed link in the 3-sphere such that Dehn surgery on this framed link gives the twisted face-pairing manifold. The components of the framed link fall naturally into two families; each curve in one family corresponds to a face pair and has framing 0, and each curve in the other family corresponds to an edge cycle and has framing the sum of the reciprocal of its multiplier and the blackboard framing of a certain projection of the curve. These results are very useful for understanding both specific face-pairing manifolds and entire classes of examples. While we defer most illustrations of these results to a later paper [4], we give several examples here to illustrate how to use these results to give familiar names to some twisted face-pairing 3-manifolds.

One of our most interesting results in this paper is that all Heegaard diagrams have a natural decomposition into subdiagrams called Heegaard cylinders, each of which has a natural shape given by the ratio of two positive integers. We characterize the Heegaard diagrams arising naturally from a twisted face-pairing description as those whose Heegaard cylinders all have integral shape. 


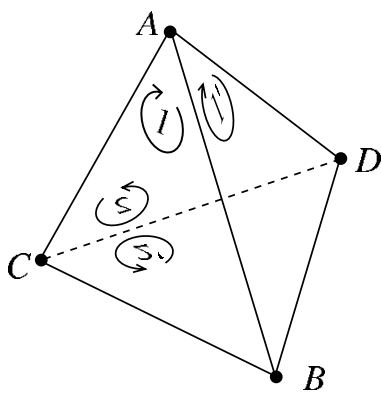

Figure 1: The complex $P$

We give a preliminary example to illustrate the twisted face-pairing construction. Let $P$ be a tetrahedron with vertices $A, B, C$, and $D$, as shown in Figure 1. Consider the face-pairing $\epsilon=\left\{\epsilon_{1}, \epsilon_{2}\right\}$ on $P$ with map $\epsilon_{1}$ which takes triangle $A B C$ to triangle $A B D$ fixing the edge $A B$ and map $\epsilon_{2}$ which takes triangle $A C D$ to $B C D$ fixing the edge $C D$. This example was considered briefly in [1] and in more detail in [2, Example 3.2]. The edge cycles are the equivalence classes of the edges of $P$ under the face-pairing maps. The three edge cycles are $\{A B\},\{B C, B D, A D, A C\}$, and $\{C D\}$; the associated diagrams of face-pairing maps are shown below.

$$
\begin{gathered}
A B \stackrel{\epsilon_{1}}{\longrightarrow} A B \\
B C \stackrel{\epsilon_{1}}{\longrightarrow} B D \stackrel{\epsilon_{2}^{-1}}{\longrightarrow} A D \stackrel{\epsilon_{1}^{-1}}{\longrightarrow} A C \stackrel{\epsilon_{2}}{\longrightarrow} B C \\
C D \stackrel{\epsilon_{2}}{\longrightarrow} C D
\end{gathered}
$$

To construct a twisted face-pairing manifold from $P$, for each edge cycle $[e]$ we choose a positive integer $\operatorname{mul}([e])$ called the multiplier of $[e]$. Let $Q$ be the subdivision of $P$ obtained by subdividing each edge $e$ of $P$ into \#([e]) . $\operatorname{mul}([e])$ subedges. The face-pairing maps $\epsilon_{1}$ and $\epsilon_{2}$ naturally give face-pairing maps on the faces of $Q$. Choose an orientation of $\partial Q$, and define the twisted face-pairing $\delta$ on $Q$ by precomposing each $\epsilon_{i}$ with an orientation-preserving homeomorphism of its domain which takes each vertex to the vertex that follows it in the induced orientation on the boundary. By the fundamental theorem of twisted face-pairings (see [1] or [2]), the quotient $Q / \delta$ is a closed 3-manifold.

To construct a Heegaard diagram and framed link for the twisted face-pairing manifold $Q / \delta$, we first schematically indicate the edge diagrams as shown in Figure 2. We then make rectangles out of the edge diagrams in Figure 3, and add thin horizontal and vertical line segments through the midpoints of each of 


$$
\left.\left.\right|_{B} ^{A} \stackrel{A}{\rightarrow}\right|_{B} ^{\varepsilon_{1}}
$$

$$
\left.\left.\left.\left.\left.\right|_{C} ^{B} \stackrel{\varepsilon_{1}}{\rightarrow}\right|_{D} ^{B} \stackrel{\varepsilon_{2}^{-l}}{\rightarrow}\right|_{D} ^{A} \stackrel{\varepsilon_{1}^{-1}}{\rightarrow}\right|_{C} ^{A} \stackrel{\varepsilon_{2}}{\rightarrow}\right|_{C} ^{B}
$$$$
\left.\left.\right|_{D} ^{\varepsilon_{2}}\right|_{D} ^{C}
$$

Figure 2: The edge diagrams
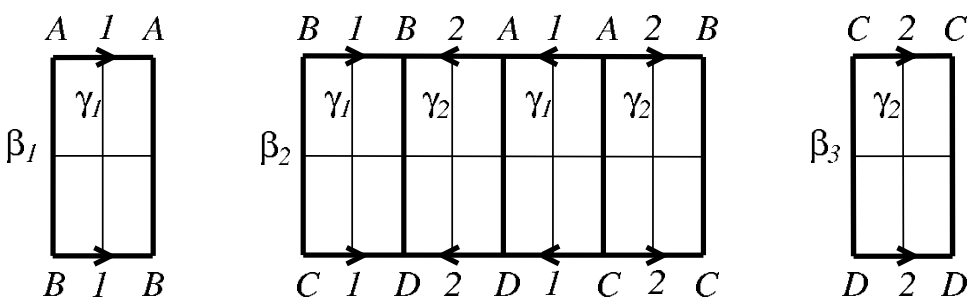

Figure 3: The rectangles that correspond to the edge diagrams

the subrectangles of the rectangles. We identify the boundary edges of the rectangles in pairs preserving the vertex labels (and, for horizontal edges, the order) to get a quotient surface $S$ of genus two. The image in $S$ of the thin vertical arcs is a union of two disjoint simple closed curves $\gamma_{1}$ and $\gamma_{2}$, which correspond to the two face pairs. The image in $S$ of the thin horizontal arcs is a union of three pairwise disjoint simple closed curves $\beta_{1}, \beta_{2}$, and $\beta_{3}$, which correspond to the three edge cycles. Figure 4 shows $S$ as the quotient of the union of two annuli, and Figure 5 shows the curve families $\left\{\gamma_{1}, \gamma_{2}\right\}$ and $\left\{\beta_{1}, \beta_{2}, \beta_{3}\right\}$ on $S$. For $i \in\{1,2,3\}$, let $m_{i}$ be the multiplier of the edge cycle corresponding to $\beta_{i}$ and let $\tau_{i}$ be one of the two Dehn twists along $\beta_{i}$. We choose $\tau_{1}, \tau_{2}$, and $\tau_{3}$ so that they are oriented consistently. Let $\tau=\tau_{1}^{m_{1}} \circ \tau_{2}^{m_{2}} \circ \tau_{3}^{m_{3}}$. It follows from Theorem 6.1.1 that $S$ and $\left\{\gamma_{1}, \gamma_{2}\right\}$ and $\left\{\tau\left(\gamma_{1}\right), \tau\left(\gamma_{2}\right)\right\}$ form a Heegaard diagram for the twisted face-pairing manifold $Q / \delta$. From the Heegaard diagram, one can use standard techniques to give a framed surgery description for $Q / \delta$. An algorithmic description for this is given in Theorem 6.1.2. In the present example, the surgery description is shown in Figure 6 together with a modification of the 1-skeleton of the tetrahedron $P$. There are two curves with framing 0 , corresponding to the two pairs of faces. The other three curves correspond to the edge cycles and have framings the reciprocals of the multipliers.

We now describe our Heegaard diagram construction in greater detail. We use the notation and terminology of [2]. Let $P$ be a faceted 3-ball, let $\epsilon$ be an orientation-reversing face-pairing on $P$, and let mul be a multiplier function 

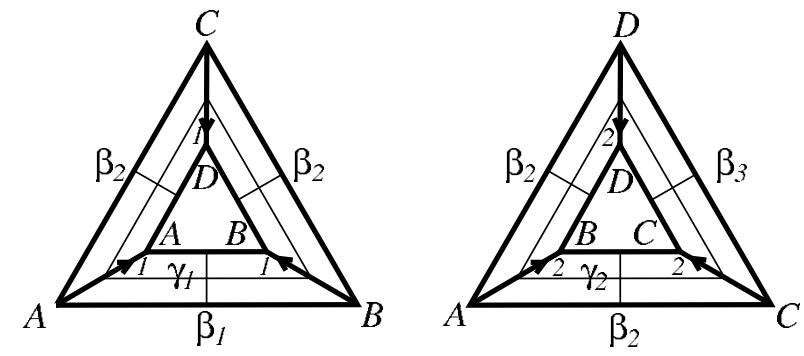

Figure 4: Another view of the surface $S$

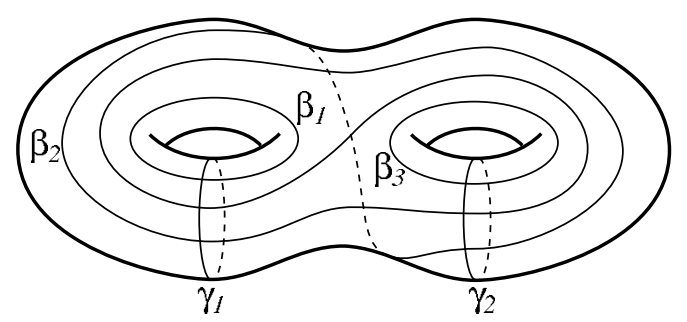

Figure 5: The curve families $\left\{\gamma_{1}, \gamma_{2}\right\}$ and $\left\{\beta_{1}, \beta_{2}, \beta_{3}\right\}$ on the surface $S$

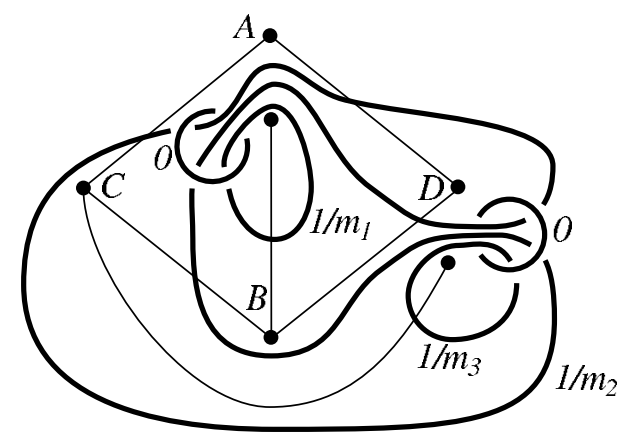

Figure 6: The surgery description

for $\epsilon$. (As in [2], we for now assume that $P$ is a regular CW complex. We drop the regularity assumption in Section 2.) Let $Q$ be the twisted face-pairing subdivision of $P$, let $\delta$ be the twisted face-pairing on $Q$, and let $M$ be the associated twisted face-pairing manifold. We next construct a closed surface $S$ with the structure of a cell complex. For this we fix a cell complex $X$ cellularly homeomorphic to the 1-skeleton of $Q$. Suppose given two paired faces $f$ and $f^{-1}$ of $Q$. We choose one of these faces, say $f$, and we construct $\partial f \times[0,1]$. We view the interval $[0,1]$ as a 1 -cell, and we view $\partial f \times[0,1]$ as a 2 -complex 
with the product cell structure. For every $x \in \partial f$ we identify $(x, 0) \in \partial f \times[0,1]$ with the point in $X$ corresponding to $x$ and we identify $(x, 1) \in \partial f \times[0,1]$ with the point in $X$ corresponding to $\delta_{f}(x) \in \partial f^{-1}$. Doing this for every pair of faces of $Q$ yields a cell complex $Y$ on a closed surface. We define $S$ to be the first dual cap subdivision of $Y$; because every face of $Y$ is a quadrilateral, this simply means that to obtain $S$ from $Y$ we subdivide every face of $Y$ into four quadrilaterals in the straightforward way. We say that an edge of $S$ is vertical if it is either contained in $X$ or is disjoint from $X$. We say that an edge of $S$ is diagonal if it is not vertical. The union of the vertical edges of $S$ which are not edges of $Y$ is a family of simple closed curves in $S$. Likewise the union of the diagonal edges of $S$ which are not edges of $Y$ is a family of simple closed curves in $S$. Theorem 4.3.1 states that the surface $S$ and these two families of curves form a Heegaard diagram for $M$.

In this paragraph we indicate how to associate to a given edge cycle $E$ of $\epsilon$ a closed subspace of $S$. To simplify this discussion we assume that $E$ contains three edges and that $\operatorname{mul}(E)=2$. When constructing $Q$ from $P$, every edge of $E$ is subdivided into $2 \cdot 3=6$ subedges. So corresponding to the three edges of $E$, the complex $S$ contains three 1-complexes, each of them homeomorphic to an interval and the union of 12 vertical edges of $S$. These three 1-complexes and part of $S$ are shown in Figure 7; the three 1-complexes are drawn as four thick vertical line segments with the left one to be identified with the right one. We refer to the closed subspace $C$ of $S$ shown in Figure 7 as an edge cycle cylinder or simply as a cylinder. In Figure 7, vertical edges of $S$ are drawn vertically and diagonal edges of $S$ are drawn diagonally. Some arcs in Figure 7 are dashed because they are not contained in the 1-skeleton of $S$. The thick edges in Figure 7 are the edges of $Y$ in $C$. (It is interesting to note that these thick edges essentially give the diagram in Figure 11 of [2].) Note that the edge cycle cylinder $C$ need not be a closed annulus, although $C$ is the closure of an open annulus. (Identifications of boundary points are possible.) We choose these edge cycle cylinders so that their union is $S$ and the cylinders of distinct $\epsilon$-edge cycles have disjoint interiors.

We define the circumference of an edge cycle cylinder to be the number of edges in its edge cycle. We define the height of an edge cycle cylinder to be the number of edges in its edge cycle times the multiplier of its edge cycle. The edge cycle cylinder $C$ in Figure 7 contains three $\operatorname{arcs} \rho_{1}, \rho_{2}, \rho_{3}$ whose endpoints lie on dashed arcs such that each of $\rho_{1}, \rho_{2}, \rho_{3}$ is a union of thin vertical edges. Likewise $C$ contains three $\operatorname{arcs} \sigma_{1}, \sigma_{2}, \sigma_{3}$ such that each of $\sigma_{1}, \sigma_{2}, \sigma_{3}$ is a union of thin diagonal edges and the endpoints of $\sigma_{i}$ equal the endpoints of $\rho_{i}$ for every $i \in\{1,2,3\}$. Because the height of $C$ equals 2 times the circumference 


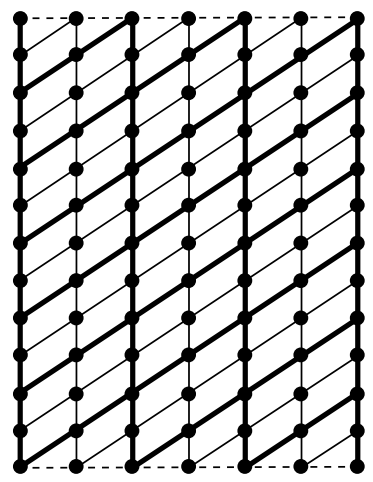

Figure 7: The cylinder $C$ corresponding to the edge cycle $E$

of $C$, it follows that $\sigma_{1}, \sigma_{2}, \sigma_{3}$ can be realized as the images of $\rho_{1}, \rho_{2}, \rho_{3}$ under the second power of a Dehn twist along a waist of $C$. This observation and the previous paragraphs essentially give the following. Let $\alpha_{1}, \ldots, \alpha_{n}$ be the simple closed curves in $S$ which are unions of vertical edges of $S$ that are not edges of $Y$. Let $E_{1}, \ldots, E_{m}$ be the edge cycles of $\epsilon$. For every $i \in\{1, \ldots, m\}$ construct a waist $\beta_{i}$ in the edge cycle cylinder of $E_{i}$ so that $\beta_{1}, \ldots, \beta_{m}$ are pairwise disjoint simple closed curves in $S$. For every $i \in\{1, \ldots, m\}$ let $\tau_{i}$ be one of the two Dehn twists on $S$ along $\beta_{i}$, chosen so that the directions in which we twist are consistent. Set $\tau^{\mathrm{mul}}=\tau_{1}^{\operatorname{mul}\left(E_{1}\right)} \circ \cdots \circ \tau_{m}^{\operatorname{mul}\left(E_{m}\right)}$. Then $S$ and $\alpha_{1}, \ldots, \alpha_{n}$ and $\tau^{\mathrm{mul}}\left(\alpha_{1}\right), \ldots, \tau^{\mathrm{mul}}\left(\alpha_{n}\right)$ form a Heegaard diagram for $M$. The last statement is the content of Theorem 6.1.1.

The result of the previous paragraph leads to a link $L$ in $S^{3}$ that has components $\gamma_{1}, \ldots, \gamma_{n}$ and $\delta_{1}, \ldots, \delta_{m}$, where $\gamma_{1}, \ldots, \gamma_{n}$ correspond to $\alpha_{1}, \ldots, \alpha_{n}$ and $\delta_{1}, \ldots, \delta_{m}$ correspond to $\beta_{1}, \ldots, \beta_{m}$. We define a framing of $L$ so that $\gamma_{1}, \ldots, \gamma_{n}$ have framing 0 and for every $i \in\{1, \ldots, m\} \delta_{i}$ has framing $\operatorname{mul}\left(E_{i}\right)^{-1}$ plus the blackboard framing of $\delta_{i}$ relative to a certain projection. Then the manifold obtained by Dehn surgery on $L$ is homeomorphic to $M$. The last statement is the content of Theorem 6.1.2. At last we see that multipliers of edge cycles are essentially inverses of framings of link components. In Section 6.2 we make the construction of $L$ algorithmic and simple using what we call the corridor construction.

We know of no nice characterization of twisted face-pairing 3-manifolds. However, Theorem 5.3.1 gives such a characterization of their Heegaard diagrams. Theorem 5.3.1 and results leading to it give the following statements. Every irreducible Heegaard diagram for an orientable closed 3-manifold $M$ gives rise to a faceted 3-ball $P$ with orientation-reversing face-pairing $\epsilon$ (in essentially 
two ways - one for each family of meridian curves) such that $P / \epsilon$ is homeomorphic to $M$. Every irreducible Heegaard diagram can be decomposed into cylinders, which we call Heegaard cylinders, essentially just as our above Heegaard diagrams of twisted face-pairing manifolds are decomposed into edge cycle cylinders. In general heights of Heegaard cylinders are not multiples of their circumferences. A given irreducible Heegaard diagram is the Heegaard diagram, as constructed above, of a twisted face-pairing manifold if and only if the height of each of its Heegaard cylinders is a multiple of its circumference. Furthermore, if the height of every Heegaard cylinder is a multiple of its circumference, then the face-pairing $\epsilon$ constructed from the given Heegaard diagram is a twisted face-pairing.

Thus far we have discussed the construction of Heegaard diagrams for twisted face-pairing manifolds and the construction of face-pairings from irreducible Heegaard diagrams. In Theorem 4.2.1 we more generally construct (irreducible) Heegaard diagrams for manifolds of the form $P / \epsilon$, where $P$ is a faceted 3-ball with orientation-reversing face-pairing $\epsilon$ and the cell complex $P / \epsilon$ is a manifold with one vertex. In Theorem 5.3.1 we construct for every irreducible Heegaard diagram for a 3-manifold $M$ a faceted 3-ball $P$ with orientation-reversing facepairing $\epsilon$ (in essentially two ways - one for each family of meridian curves) such that $P / \epsilon$ is a cell complex with one vertex and $P / \epsilon$ is homeomorphic to $M$. These two constructions are essentially inverse to each other.

The above statements that every irreducible Heegaard diagram gives rise to a faceted 3-ball require a more general definition of faceted 3-ball than the one given in [2]. In [2] faceted 3-balls are regular, that is, for every open cell of a faceted 3-ball the prescribed homeomorphism of an open Euclidean ball to that cell extends to a homeomorphism of the closed Euclidean ball to the closed cell. On the other hand, the cellulation of the boundary of a 3-ball which arises from a Heegaard diagram has paired faces but otherwise is arbitrary. So we now define a faceted 3-ball $P$ to be an oriented $\mathrm{CW}$ complex such that $P$ is a closed 3 -ball, the interior of $P$ is the unique open 3-cell of $P$, and the cell structure of $\partial P$ does not consist of just one 0 -cell and one 2-cell. This generalization presents troublesome minor technical difficulties but no essential difficulties. In particular, all the results of [1] and [2] hold for these more general faceted 3balls. Section 2 deals with this generalization. Except when the old definition is explicitly discussed, we henceforth in this paper use the new definition of faceted 3-ball. We know of no reducible twisted face-pairing manifold which arises from a regular faceted 3-ball; the old twisted face-pairing manifolds all seem to be irreducible. On the other hand the new twisted face-pairing manifolds are often reducible. See Examples 2.1 (which is considered again in 4.3.2 and 7.1) and 
2.3 (which is considered again in 6.2.1).

Our construction of Heegaard diagrams from face-pairings uses a subdivision of cell complexes which we call dual cap subdivision. We define and discuss dual cap subdivision in Section 3. The term "dual" is motivated by the notion of dual cell complex, and the term "cap" is motivated by its association with intersection. Intuitively, the dual cap subdivision of a cell complex is gotten by "intersecting" the complex with its "dual complex". Dual cap subdivision is coarser than barycentric subdivision, and it is well suited to the constructions at hand. Heegaard decompositions of 3-manifolds are usually constructed by triangulating the manifolds and working with their second barycentric subdivisions. Instead of using barycentric subdivision, we use dual cap subdivision, and we obtain the following. Earlier in the introduction we constructed a surface $S$ with a cell structure. We show that $S$ is cellularly homeomorphic to a subcomplex of the second dual cap subdivision of the manifold $M$, where this subcomplex corresponds to the usual Heegaard surface gotten by using a triangulation and barycentric subdivision.

In Section 7 we use the corridor construction of Section 6.2 to construct links in $S^{3}$ for three different model face-pairings. Simplifying these links using isotopies and Kirby calculus, we are able to identify the corresponding twisted face-pairing manifolds. In Example 7.1 we obtain the connected sum of the lens space $L(p, 1)$ and the lens space $L(r, 1)$ as a twisted face-pairing manifold, where $p$ and $r$ are positive integers. In Example 7.2 we obtain all integer Dehn surgeries on the figure eight knot as twisted face-pairing manifolds. In Example 7.3 we obtain the Heisenberg manifold, the prototype of Nil geometry. In Example 6.2.1 we obtain $S^{2} \times S^{1}$.

Which orientable connected closed 3-manifolds are twisted face-pairing manifolds? As far as we know they all are, although that seems rather unlikely. An interesting problem is to determine whether the 3-torus is a twisted face-pairing manifold; we do not know whether it is or not. In a later paper [4] we present a survey of twisted face-pairing 3-manifolds which indicates the scope of the set of twisted face-pairing manifolds. Here are some of the results in [4]. We show how to obtain every lens space as a twisted face-pairing manifold. We consider the faceted 3-balls for which every face is a digon, and we show that the twisted face-pairing manifolds obtained from these faceted 3-balls are Seifert fibered manifolds. We show how to obtain most Seifert fibered manifolds. We show that if $M_{1}$ and $M_{2}$ are twisted face-pairing manifolds, then so is the connected sum of $M_{1}$ and $M_{2}$.

This research was supported in part by National Science Foundation grants 
DMS-9803868, DMS-9971783, and DMS-10104030. We thank the referee for helpful suggestions on improving the exposition.

\section{Generalizing the construction}

Our twisted face-pairing construction begins with a faceted 3-ball. In Section 2 of [2] we define a faceted 3-ball $P$ to be an oriented regular CW complex such that $P$ is a closed 3 -ball and $P$ has a single 3 -cell. In this section we generalize our twisted face-pairing construction by generalizing the notion of faceted 3-ball. This generalization gives us more freedom in constructing twisted face-pairing manifolds, and it is natural in the context of Theorem 5.3.1.

We take cells of cell complexes to be closed unless explicitly stated otherwise.

We now define a faceted 3 -ball $P$ to be an oriented $\mathrm{CW}$ complex such that $P$ is a closed 3 -ball, the interior of $P$ is the unique open 3-cell of $P$, and the cell structure of $\partial P$ does not consist of just one 0-cell and one 2-cell. Suppose that $P$ is an oriented CW complex such that $P$ is a closed 3-ball and the interior of $P$ is the unique open 3-cell of $P$. The condition that the cell structure of $\partial P$ does not consist of just one 0 -cell and one 2-cell is equivalent to the following useful condition. For every 2-cell $f$ of $P$ there exists a CW complex $F$ such that $F$ is a closed disk, the interior of $F$ is the unique open 2-cell of $F$, and there exists a continuous cellular map $\varphi: F \rightarrow f$ such that the restriction of $\varphi$ to every open cell of $F$ is a homeomorphism. So $f$ is gotten from $F$ by identifying some vertices and identifying some pairs of edges. The number of vertices and edges in $F$ is uniquely determined. This definition of faceted 3-ball allows for faces such as those in Figure 8, which were not allowed before; part a) of Figure 8 shows a quadrilateral and part b) of Figure 8 shows a pentagon. To overcome difficulties presented by faces such as those in Figure 8, the next thing that we do is subdivide $P$.

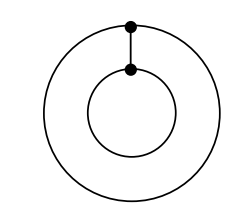

a)

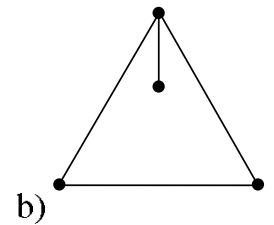

b)

Figure 8: Faces now allowed in a faceted 3-ball

In this paragraph we construct a subdivision $P_{s}$ of a given faceted 3-ball $P$. The idea is to not subdivide the 3-cell of $P$ and to construct what might be

Algebraic 6 Geometric Topology, Volume 3 (2003) 
called the barycentric subdivision of $\partial P$. The vertices of $P_{s}$ are the vertices of $P$ together with a barycenter for every edge of $P$ and a barycenter for every face of $P$. Every face of $P_{s}$ is a triangle contained in $\partial P$. If $t$ is one of these triangles, then one vertex of $t$ is a vertex of $P$, one vertex of $t$ is a barycenter of an edge of $P$, and one vertex of $t$ is a barycenter of a face of $P$. The only 3 -cell of $P_{s}$ is the 3-cell of $P$. This determines $P_{s}$. Given a face $f$ of $P$, we let $f_{s}$ denote the subcomplex of $P_{s}$ which consists of the cells of $P_{s}$ contained in $f$. Figure 9 shows $f_{s}$ for each of the faces $f$ in Figure 8 .
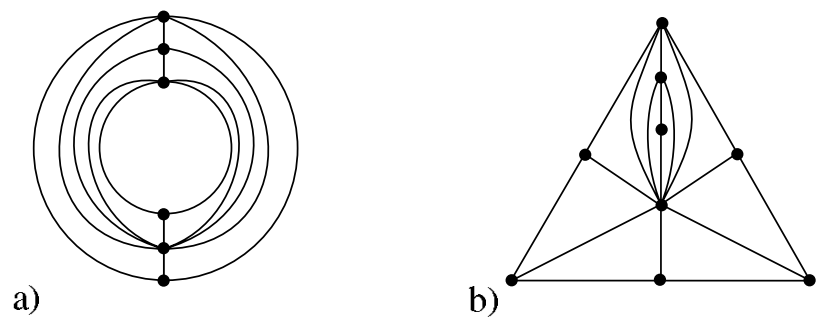

Figure 9: The subdivisions of the faces in Figure 8

In this paragraph we make two related definitions. Let $P$ be a faceted 3 -ball, and let $f$ be a face of $P$. We define a corner of $f$ at a vertex $v$ of $f$ to be a subcomplex of $f_{s}$ consisting of the union of two faces of $f_{s}$ which both contain an edge $e$ such that $e$ contains $v$ and the barycenter of $f$. We define an edge cone of $f$ at an edge $e$ of $f$ to be a subcomplex of $f_{s}$ consisting of the union of two faces of $f_{s}$ which both contain an edge $e^{\prime}$ such that $e^{\prime}$ contains the barycenter of $f$ and the barycenter of $e$.

A face-pairing $\epsilon$ on a given faceted 3-ball $P$ now consists of the following. First, the faces of $P$ are paired: for every face $f$ of $P$ there exists a face $f^{-1} \neq f$ of $P$ such that $\left(f^{-1}\right)^{-1}=f$. Second, the faces of $P_{s}$ are paired: for every face $t$ of $P_{s}$ contained in a face $f$ of $P$ there exists a face $t^{-1}$ of $P_{s}$ with $t^{-1} \subseteq f^{-1}$ such that $\left(t^{-1}\right)^{-1}=t$. Third, for every face $t$ of $P_{s}$ there exists a cellular homeomorphism $\epsilon_{t}: t \rightarrow t^{-1}$ called a partial face-pairing map such that $\epsilon_{t^{-1}}=\epsilon_{t}^{-1}$. We require that $\epsilon_{t}$ maps the vertex of $P$ in $t$ to the vertex of $P$ in $t^{-1}$, that $\epsilon_{t}$ maps the edge barycenter in $t$ to the edge barycenter in $t^{-1}$, and that $\epsilon_{t}$ maps the face barycenter in $t$ to the face barycenter in $t^{-1}$. Furthermore, the faces of $P_{s}$ are paired and the partial face-pairing maps are defined so that if $t$ and $t^{\prime}$ are faces of $P_{s}$ contained in some face $f$ of $P$ and if $e$ is an edge of $t \cap t^{\prime}$ which contains the barycenter of $f$, then $\left.\epsilon_{t}\right|_{e}=\left.\epsilon_{t^{\prime}}\right|_{e}$. For every face $f$ of $P$ we set $\epsilon_{f}=\left\{\epsilon_{t}: t\right.$ is a face of $\left.f_{s}\right\}$, and we refer to $\epsilon_{f}$ as a multivalued face-pairing map from $f$ to $f^{-1}$. We set $\epsilon=\left\{\epsilon_{f}: f\right.$ is a face of $\left.P\right\}$. 
In a straightforward way we obtain a quotient space $P_{s} / \epsilon$ consisting of orbits of points of $P_{s}$ under $\epsilon$. Finally, as in [2, Section 2] we impose on $\epsilon$ the face-pairing compatibility condition that as one goes through an edge cycle the composition of face-pairing maps is the identity. The cell structure of $P$ induces a cell structure on $P_{s} / \epsilon$, and it is this cell structure that we put on $P_{s} / \epsilon$, not the cell structure induced from $P_{s}$. We usually write $P / \epsilon$ instead of $P_{s} / \epsilon$. We usually want $\epsilon$ to be orientation reversing, which means that every partial face-pairing map of $\epsilon$ reverses orientation.

Let $P$ be a faceted 3-ball, let $f$ be a face of $P$, and suppose that $\epsilon$ is an orientation-reversing face-pairing on $P$. Then the multivalued face-pairing map $\epsilon_{f}$ determines a function from the set of corners of $f$ to the set of corners of $f^{-1}$ in a straightforward way. The image of one corner of $f$ under this function determines the image of every corner of $f$ under this function. The action of $\epsilon$ on the set of corners of the faces of $P$ determines $P_{s} / \epsilon$ up to homeomorphism. Thus for our purposes to define the multivalued face-pairing map $\epsilon_{f}$ of a face $f$ of $P$, it suffices to give a corner $c$ of $f$ and the corner of $f^{-1}$ to which $\epsilon_{f}$ maps $c$.

Let $\epsilon$ be an orientation-reversing face-pairing on a faceted 3-ball $P$. Essentially as in Section 2 of [2], $\epsilon$ partitions the edges of $P$ into edge cycles. (We consider the edges of $P$, not the edges of $P_{s}$.) To every edge cycle $E$ of $\epsilon$ we associate a length $\ell_{E}$ and a multiplier $m_{E}$ as before. The function mul: \{edge cycles\} $\rightarrow$ $\mathbf{N}$ defined by $E \mapsto m_{E}$ is called the multiplier function. We obtain a twisted face-pairing subdivision $Q$ from $P$ just as before: if $e$ is an edge of $P$ and if $E$ is the edge cycle of $\epsilon$ containing $e$, then we subdivide $e$ into $\ell_{E} m_{E}$ subedges. As before, we subdivide in an $\epsilon$-invariant way. We likewise construct $Q_{s}$ in an $\epsilon$-invariant way. It follows that $\epsilon$ naturally determines a face-pairing on $Q$, which we continue to call $\epsilon$, abusing notation more than before.

We consider face twists in this paragraph. In the present setting a face twist is not a single cellular homeomorphism, but instead a collection of cellular homeomorphisms. For this, we maintain the situation of the previous paragraph. Let $f$ be a face of $Q$. Let $t$ be a face of $f_{s}$. The orientation of $f$ determines a cyclic order on the faces of $f_{s}$. Let $t^{\prime}$ be the second face of $f_{s}$ which follows $t$ relative to this cyclic order. Let $\tau_{t}$ be an orientation-preserving cellular homeomorphism from $t$ to $t^{\prime}$ such that $\tau_{t}$ fixes the barycenter of $f$. We call $\tau_{f}=\left\{\tau_{t}: t\right.$ is a face of $\left.f_{s}\right\}$ the face twist of $f$. We assume that if $t_{1}$ and $t_{2}$ are faces of $f_{s}$ and if $e$ is an edge of $t_{1} \cap t_{2}$ which contains the barycenter of $f$, then $\left.\tau_{t_{1}}\right|_{e}=\left.\tau_{t_{2}}\right|_{e}$. We also assume that our face twists are defined $\epsilon$-invariantly: for each face $f$ of $Q$ and each face $t$ of $f_{s}$, we have $\tau_{t^{-1}}=\epsilon_{t^{\prime \prime}} \circ \tau_{t^{\prime \prime}}^{-1} \circ \epsilon_{t^{-1}}$, 
where $t^{\prime \prime}$ is the second face of $f_{s}$ which precedes $t$. We furthermore impose a compatibility condition on our face twists in the next paragraph.

Now we are prepared to define a twisted face-pairing $\delta$ on $Q$. We pair the faces of $Q$ just as the faces of $P$ are paired. The pairing of the faces of $P_{s}$ likewise induces a pairing of the faces of $Q_{s}$. For every face $f$ of $Q$ and every face $t$ of $f_{s}$, we set $\delta_{t}=\epsilon_{t^{\prime}} \circ \tau_{t}$, where $t^{\prime}$ is the second face of $f_{s}$ which follows $t$. For every face $f$ of $Q$ we set $\delta_{f}=\left\{\delta_{t}: t\right.$ is a face of $\left.f_{s}\right\}$, and we set $\delta=\left\{\delta_{f}: f\right.$ is a face of $\left.Q\right\}$. We assume that the maps $\tau_{t}$ are defined so that $\delta$ satisfies the face-pairing compatibility condition that as one goes through a cycle of edges in $Q$ the compositions of face-pairing maps is the identity. Then $\delta$ is a face-pairing on $Q$ called the twisted face-pairing.

Finally, we define $M=M(\epsilon$, mul $)$ to be the quotient space $Q_{s} / \delta$. We emphasize that for a cell structure on $M$ we take the cell structure induced from $Q$, not the cell structure induced from $Q_{s}$. The cell complex $M$ is determined up to homeomorphism by the function mul and the action of $\epsilon$ on the corners of the faces of $P$.

Let $P$ be a faceted 3-ball, let $\epsilon$ be an orientation-reversing face-pairing on $P$, and let mul be a multiplier function for $\epsilon$. The results of [2] all hold in this more general setting. So $M$ is an orientable closed 3-dimensional manifold with one vertex. The dual of the link of that vertex is isomorphic to $\partial Q^{*}$ as oriented 2-complexes, where $Q^{*}$ is a faceted 3-ball gotten from $Q$ by reversing orientation. We label and direct the faces and edges of $Q$ and $Q^{*}$ as before. We again obtain a duality between $M$ and $M^{*}$. The proofs in [2] are valid in the present more general setting with only straightforward minor technical modifications and the following. To obtain a duality between $M$ and $M^{*}$ in [2], we construct a dual cap subdivision $Q_{\sigma}$ of $Q$. We let $C_{1}, \ldots, C_{k}$ be the 3-cells of $Q_{\sigma}$, and for every $i \in\{1, \ldots, k\}$ we let $A_{i}$ be a cell complex isomorphic to $C_{i}$ so that $A_{1}, \ldots, A_{k}$ are pairwise disjoint. Then the vertices of $Q$ can be enumerated as $x_{1}, \ldots, x_{k}$ so that $C_{i}$ is the unique 3-cell of $Q_{\sigma}$ which contains $x_{i}$ for $i \in\{1, \ldots, k\}$. If $x_{i}$ has valence $v_{i}$, then $A_{i}$ is an alternating suspension on a $2 v_{i}$-gon for $i \in\{1, \ldots, k\}$. In the present setting the 3-cells of $Q_{\sigma}$ need not be alternating suspensions; they are quotients of alternating suspensions. See Section 3.2 for a discussion of the 3-cells of $Q_{\sigma}$. So in the present setting we let $x_{1}, \ldots, x_{k}$ be the vertices of $Q$ with valences $v_{1}, \ldots, v_{k}$, and for $i \in\{1, \ldots, k\}$ we simply define $A_{i}$ to be an alternating suspension on a $2 v_{i}$-gon. As in [2] the twisted face-pairing $\delta$ on $Q$ induces in a straightforward way what might be called a face-pairing on the disjoint union of $A_{1}, \ldots, A_{k}$. At this point we proceed as in [2]. 
We conclude this section with two simple examples to illustrate some of the new phenomena which occur for our more general faceted 3-balls.

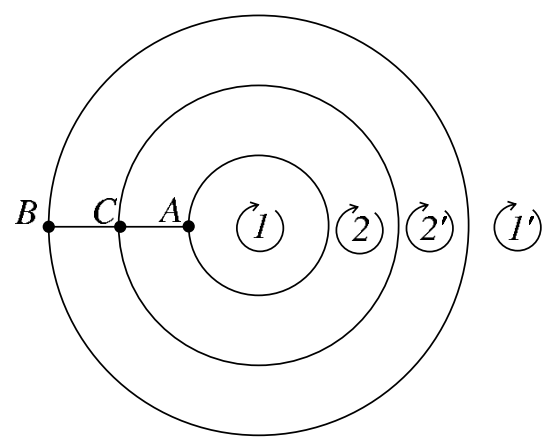

Figure 10: The complex $P$ for Example 2.1

Example 2.1 Let the model faceted 3-ball $P$ be as indicated in Figure 10 with two monogons and two quadrilaterals, the outer monogon being at infinity. The inner monogon has label 1 and is directed outward. The outer monogon has label 1 and is directed inward. The inner quadrilateral has label 2 and is directed outward. The outer quadrilateral has label 2 and is directed inward. As usual for faces in figures, all four faces are oriented clockwise. We construct an orientation-reversing face-pairing $\epsilon$ on $P$ as follows. Multivalued face-pairing map $\epsilon_{1}$ maps the inner monogon to the outer monogon, there being essentially only one way to do this. Multivalued face-pairing map $\epsilon_{2}$ maps the inner quadrilateral to the outer quadrilateral fixing their common edge. Set $\epsilon=$ $\left\{\epsilon_{1}^{ \pm 1}, \epsilon_{2}^{ \pm 1}\right\}$.

We might view this face-pairing as follows. Construct a monogon in the open northern hemisphere of the 2-sphere $S^{2}$, put a vertex on the equator of $S^{2}$ and join the two vertices with an edge. Now vertically project this cellular decomposition of the northern hemisphere into the southern hemisphere.

The edge cycles for $\epsilon$ have the following diagrams.

$$
C C \stackrel{\epsilon_{2}}{\longrightarrow} C C \quad A C \stackrel{\epsilon_{2}}{\longrightarrow} B C \stackrel{\epsilon_{2}^{-1}}{\longrightarrow} A C \quad B B \stackrel{\epsilon_{2}^{-1}}{\longrightarrow} A A \stackrel{\epsilon_{1}}{\longrightarrow} B B
$$

For now let the first edge cycle have multiplier 4, let the second have multiplier 1 , and let the third have multiplier 1 .

Figure 11 shows the faceted 3-ball $Q$; we labeled the new vertices of $Q$ arbitrarily. Figure 12 shows the link of the vertex of $M$, with conventions as in [2]. Figure 13 shows the faceted 3-ball $Q^{*}$ dual to $Q$ with its edge labels and 


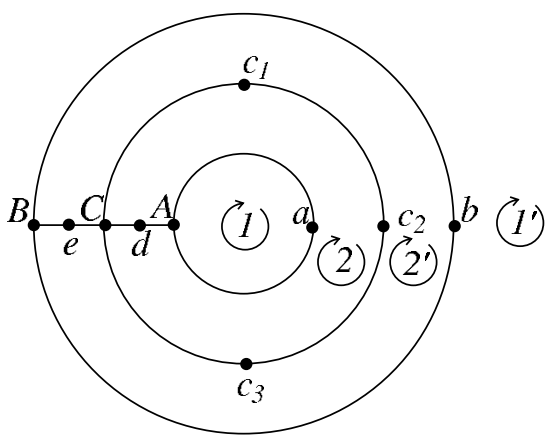

Figure 11: The complex $Q$ for Example 2.1

directions. Note that $\partial Q^{*}$ is dual to the link of the vertex of $M$. We obtain a presentation for the fundamental group $G$ of $M$ as follows. Corresponding to the face labels 1 and 2 we have generators $x_{1}$ and $x_{2}$. The boundary of the face of $Q^{*}$ labeled 1 and directed outward gives the relator $x_{1} x_{2}^{-1}$. The boundary of the face of $Q^{*}$ labeled 2 and directed outward gives the relator $x_{2}^{5} x_{1}^{-1}$. So

$$
G \cong\left\langle x_{1}, x_{2}: x_{1} x_{2}^{-1}, x_{2}^{5} x_{1}^{-1}\right\rangle \cong \mathbf{Z} / 4 \mathbf{Z} .
$$

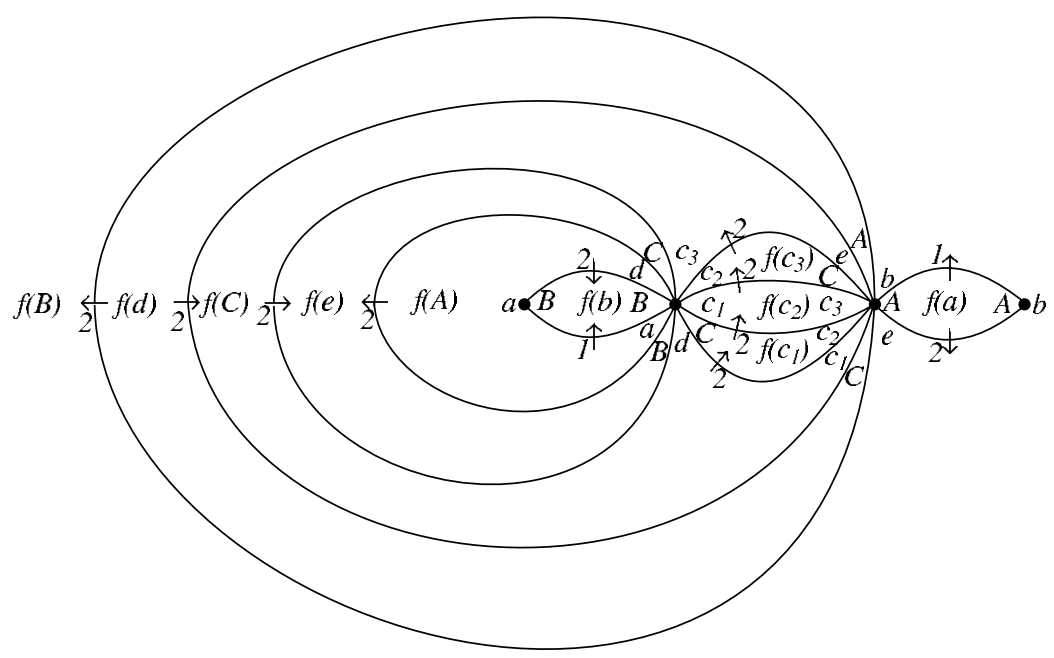

Figure 12: The link of the vertex of $M$

We will see in Example 7.1 that $M$ is the lens space $L(4,1)$. In general, if the first edge cycle of $\epsilon$ has multiplier $p$, if the second edge cycle of $\epsilon$ has multiplier $q$, and if the third edge cycle of $\epsilon$ has multiplier $r$, then we will see 


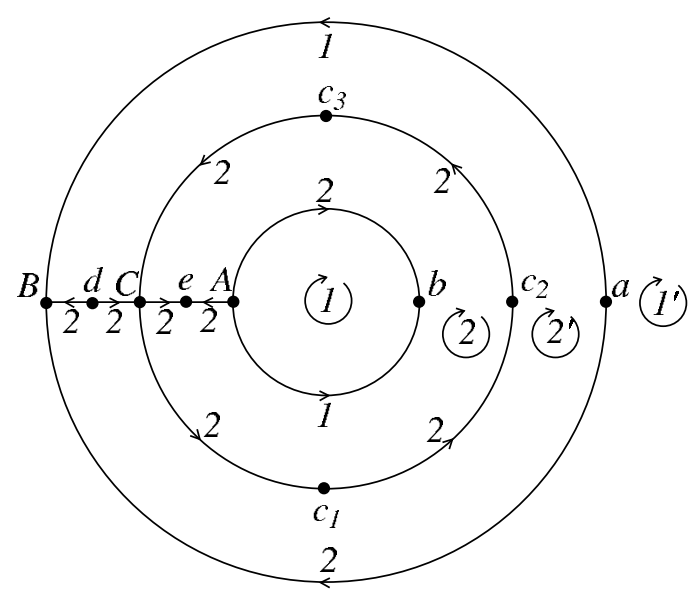

Figure 13: The complex $Q^{*}$ with edge labels and directions

in Example 7.1 that $M$ is the connected sum of the lens space $L(p, 1)$ and the lens space $L(r, 1)$ (and so in particular $M$ does not depend on $q$ ).

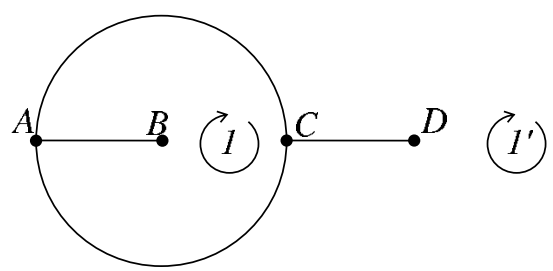

Figure 14: The complex $P$ for Example 2.3

Example 2.3 Let the model faceted 3-ball $P$ be as in Figure 14 with two quadrilaterals, the outer quadrilateral being at infinity. The inner quadrilateral has label 1 and is directed outward. The outer quadrilateral has label 1 and is directed inward. The orientation-reversing multivalued face-pairing map $\epsilon_{1}$ maps the inner quadrilateral to the outer quadrilateral taking vertex $C$ to vertex $D$. Set $\epsilon=\left\{\epsilon_{1}^{ \pm 1}\right\}$.

The vertices $A$ and $C$ of $P$ are joined by two edges. We use the subscripts $u$ and $d$ for up and down to distinguish them. So $A C_{u}$ is the upper edge joining $A$ and $C$, and $A C_{d}$ is the lower edge joining $A$ and $C$. The face-pairing $\epsilon$ has only one edge cycle, and this edge cycle has the following diagram.

$$
A C_{u} \stackrel{\epsilon_{1}}{\longrightarrow} C D \stackrel{\epsilon_{1}^{-1}}{\longrightarrow} A C_{d} \stackrel{\epsilon_{1}^{-1}}{\longrightarrow} B A \stackrel{\epsilon_{1}}{\longrightarrow} A C_{u}
$$


For simplicity let this edge cycle have multiplier 1.

Figure 15 shows the faceted 3-ball $Q$; we labeled the new vertices of $Q$ arbitrarily. Figure 16 shows the link of the vertex of $M$. Figure 17 shows the faceted 3-ball $Q^{*}$ dual to $Q$ with its edge labels and directions. Note that $\partial Q^{*}$ is dual to the link of the vertex of $M$. We obtain a presentation for the fundamental group $G$ of $M$ as follows. Corresponding to the face label 1 we have a generator $x_{1}$. The boundary of the face of $Q^{*}$ labeled 1 and directed outward gives the trivial relator. So $G$ has one generator and no relators, that is, $G \cong \mathbf{Z}$.

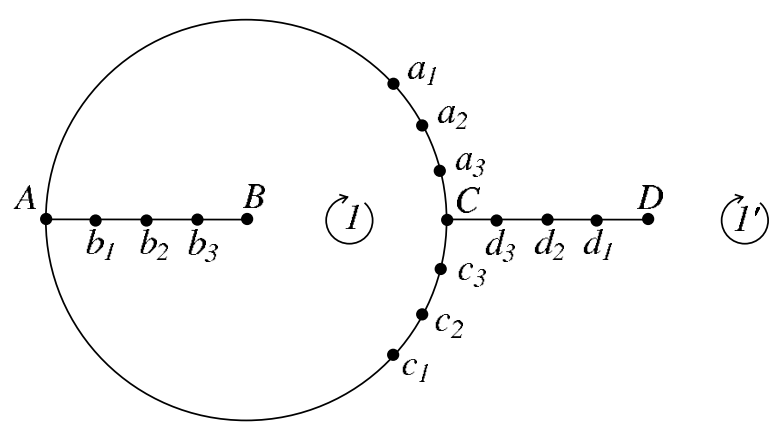

Figure 15: The complex $Q$ for Example 2.3

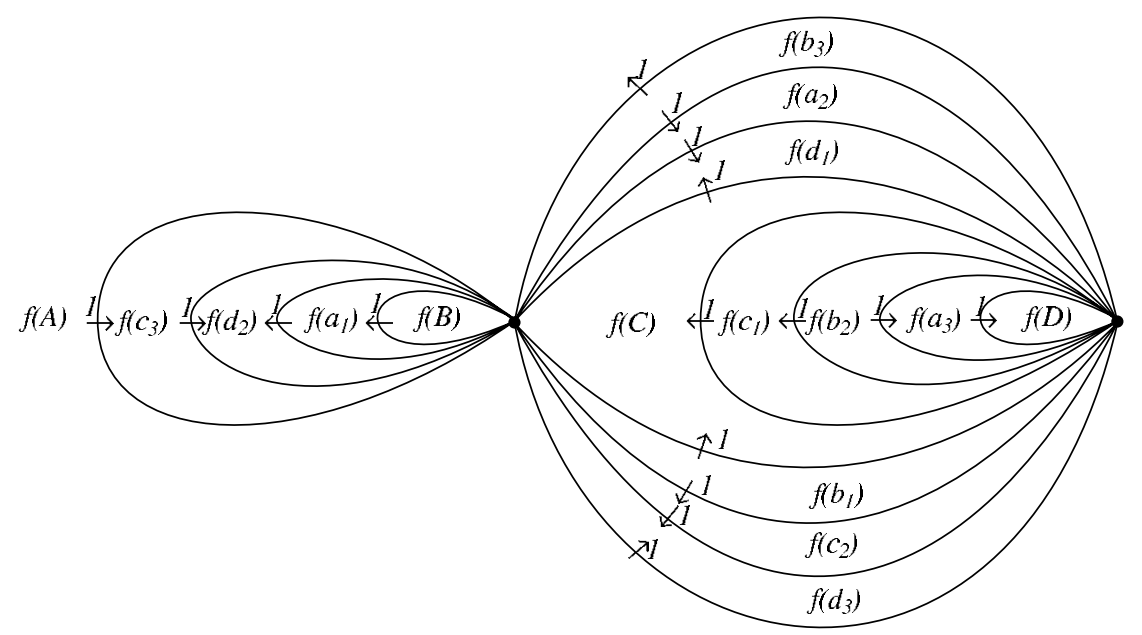

Figure 16: The link of the vertex of $M$

We will see in Example 6.2.1 that $M$ is homeomorphic to $S^{2} \times S^{1}$ for every choice of multiplier for the edge cycle of $\epsilon$. 


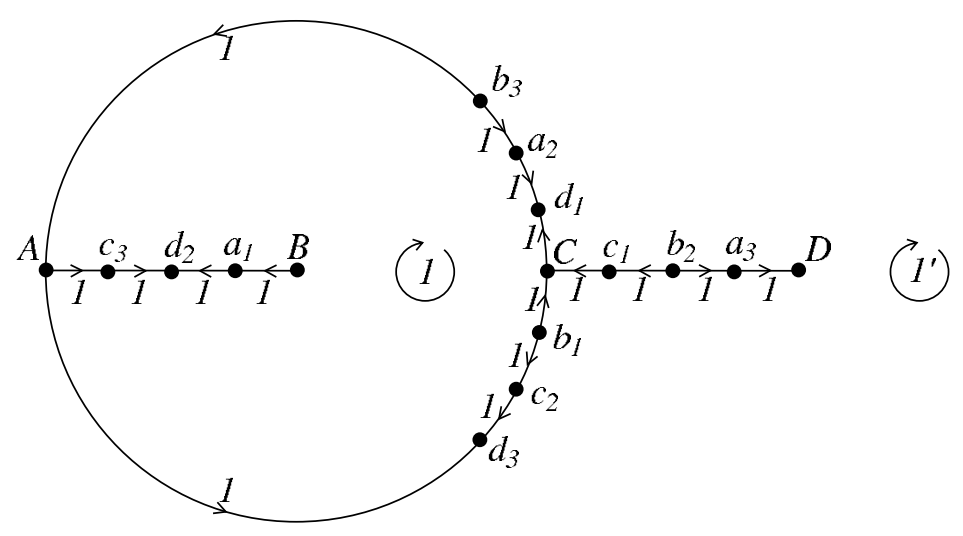

Figure 17: The complex $Q^{*}$ with edge labels and directions

\section{Dual cap subdivision}

\subsection{Definition}

Recall that we discussed dual cap subdivision in Section 4 of [2]. Of course, there our faceted 3-balls are regular. We generalize to our present cell complexes in a straightforward way.

Let $P$ be a faceted 3-ball. We construct a dual cap subdivision $P_{\sigma}$ of $P$ as follows. The vertices of $P_{\sigma}$ consist of the vertices of the subdivision $P_{s}$ defined in Section 2 together with a barycenter for the 3-cell of $P$. We next describe the edges of $P_{\sigma}$.

The edges of $\partial P_{\sigma}$ consist of the edges of $P_{s}$ which do not join the barycenter of a face of $P$ and a vertex of that face. For every face of $P$, the subdivision $P_{\sigma}$ also contains an edge joining the barycenter of that face and the barycenter of the 3-cell of $P$. These are all the edges of $P_{\sigma}$.

Having described the edges of $P_{\sigma}$, the structure of $\partial P_{\sigma}$ is determined. The faces of $\partial P_{\sigma}$ are in bijective correspondence with the corners of the faces of $P$. Every face of $\partial P_{\sigma}$ is a quadrilateral whose underlying space equals the underlying space of a corner $c$ at a vertex $v$ of a face $f$ of $P$. Of course, this quadrilateral contains the barycenter $a$ of $f$. The first diagram in Figure 18 shows this quadrilateral if $c$ has three vertices and $f$ is a monogon. The second diagram in Figure 18 shows this quadrilateral if $c$ has three vertices and $f$ is not a monogon. The third diagram in Figure 18 shows this quadrilateral if $c$ has four vertices. In the first two diagrams $b$ is the barycenter of the edge of $f$ 
that contains $v$, and in the third diagram $b_{1}$ and $b_{2}$ are the barycenters of the two edges of $f$ that contain $v$.
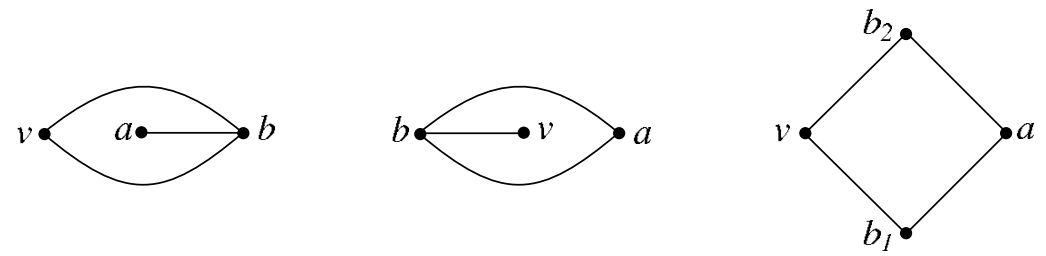

Figure 18: The three types of faces of $\partial P_{\sigma}$

The remaining faces of $P_{\sigma}$ are in bijective correspondence with the edges of $P$. Let $e$ be an edge of $P$, and let $b$ be the barycenter of $e$. We have constructed exactly two edges $e_{1}$ and $e_{2}$ in $\partial P_{\sigma}$ which contain $b$ and are not contained in $e$. The edge $e$ determines a quadrilateral face of $P_{\sigma}$ containing $e_{1} \cup e_{2}$ and the barycenter $u$ of the 3-cell of $P$. If $e$ is contained in two distinct faces of $P$, then the face of $P_{\sigma}$ determined by $e$ has four distinct edges as in the first diagram of Figure 19. If $e$ is contained in just one face of $P$, then the face of $P_{\sigma}$ determined by $e$ is a degenerate quadrilateral as in the second diagram of Figure 19. We have now described all the faces of $P_{\sigma}$. This determines $P_{\sigma}$. Note that every vertex of $P$ is in a unique 3-cell of $P_{\sigma}$.
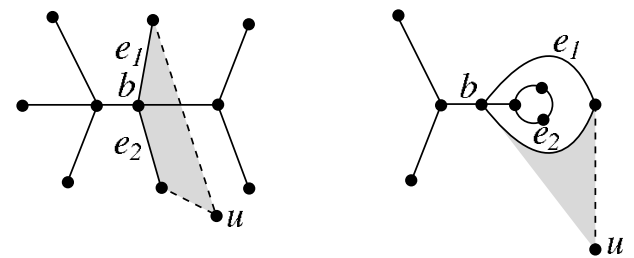

Figure 19: Faces of $P_{\sigma}$ not contained in $\partial P_{\sigma}$

Now that we have defined dual cap subdivisions of faceted 3-balls, we define dual cap subdivisions of more general cell complexes. Let $X$ be a CW complex which is the union of its 3-cells, and suppose that for every 3-cell $C$ of $X$ there exists a faceted 3-ball $B$ and a continuous cellular map $\varphi: B \rightarrow C$ such that the restriction of $\varphi$ to every open cell of $B$ is a homeomorphism. We say that a subdivision $X_{\sigma}$ of $X$ is a dual cap subdivision of $X$ if for every such choice of $C$ the cell structure on $C$ induced from $X_{\sigma}$ pulls back via $\varphi$ to give a dual cap subdivision of $B$.

It is now clear how to also define a dual cap subdivision of every CW complex with dimension at most 2 such that every 2 -cell contains an edge. If $X$ is a cell 
complex for which we have defined a dual cap subdivision and $k$ is a positive integer, then we let $X_{\sigma^{k}}$ denote the $k$-th dual cap subdivision of $X$.

\subsection{Structure of 3-cells}

In this subsection we discuss the structure of the 3-cells which occur in the dual cap subdivision of a faceted 3-ball.

Let $P$ be a regular faceted 3-ball. In Section 4 of [2] we showed that every 3 -cell of $P_{\sigma}$ is an alternating suspension. Every 3-cell of $P_{\sigma}$ contains exactly one vertex of $P$, and every vertex of $P$ is contained in exactly one 3-cell of $P_{\sigma}$. If $v$ is a vertex of $P$ with valence $k$, then the 3 -cell $B$ of $P_{\sigma}$ which contains $v$ is an alternating suspension of a $2 k$-gon. See Figure 20 , which is the same as Figure 15 of [2]. In Figure 20 the vertex $v$ is a vertex of $P$ and $u$ is the barycenter of the 3 -cell of $P$. Figure 20 shows an alternating suspension of an octagon.

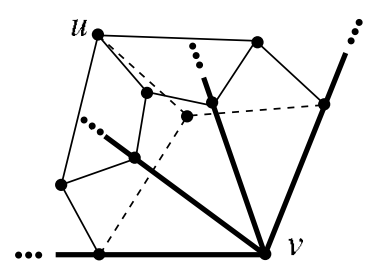

Figure 20: The 3-cell $B$ of $P_{\sigma}$ which contains the vertex $v$ of $P$

We point out here an important property of the dual cap subdivision of an alternating suspension. Let $B$ be an alternating suspension as in the previous paragraph. Because the faces of $\partial B$ are quadrilaterals and $B$ is homeomorphic to the cone on $\operatorname{star}(v, \partial B)$, the 3-cell of $B_{\sigma}$ which contains $u$ is homeomorphic to $B$ by a cellular homeomorphism $\theta: \operatorname{star}\left(u, B_{\sigma}\right) \rightarrow B$ with the following property: if $x$ is a vertex of $\operatorname{star}\left(u, B_{\sigma}\right)$ and $X$ is a cell of $B$ with $x \in X$, then $\theta(x) \in X$. Figure 21 shows $\operatorname{star}\left(u, B_{\sigma}\right)$ for the 3 -cell $B$ from Figure 20. For convenience further in this section, we make the following definition. Suppose that $V$ is a CW complex with dimension at most $3, U$ is a subcomplex of $V_{\sigma}$, and $\theta: U \rightarrow V$ is a cellular homeomorphism. We say that $\theta$ keeps vertices in their cells if $\theta(x) \in X$ whenever $x$ is a vertex of $U$ and $X$ a cell in $V$ with $x \in X$.

Now we consider the case of a general faceted 3-ball $P$. Let $v$ be a vertex of $P$. Let $e_{1}, \ldots, e_{k}$ be the edges of $P_{\sigma}$ which contain $v$. For every $i \in\{1, \ldots, k\}$ let $v_{i}$ be the vertex of $e_{i}$ unequal to $v$. There are $k$ corners of faces at $v$. Let 


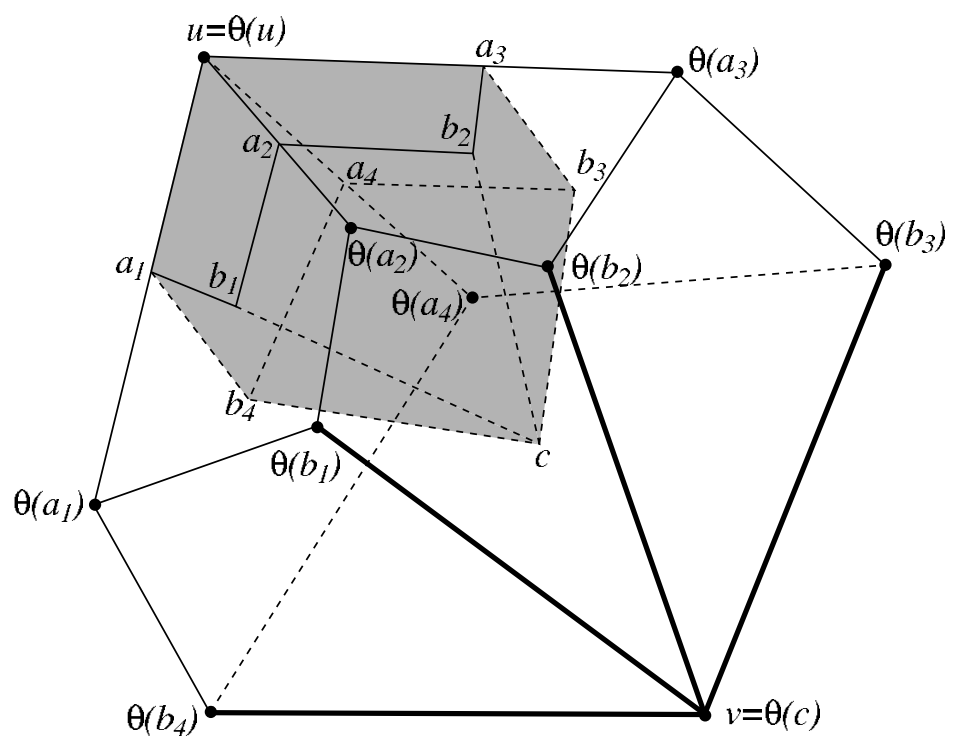

Figure 21: $\operatorname{Star}\left(u, B_{\sigma}\right)$

$f_{1}, \ldots, f_{k}$ be the faces which contain these corners. Let $u$ be the barycenter of the 3 -cell of $P$, and let $u_{i}$ be the barycenter of $f_{i}$ for every $i \in\{1, \ldots, k\}$. If $u_{1}, \ldots, u_{k}$ and $v_{1}, \ldots, v_{k}$ are distinct, then just as in the previous paragraph, there is exactly one 3 -cell of $P_{\sigma}$ which contains $v$ and this 3 -cell is an alternating suspension of a $2 k$-gon with cone points $u$ and $v$. In general exactly one 3 -cell of $P_{\sigma}$ contains $v$ and every 3 -cell of $P_{\sigma}$ contains exactly one vertex of $P$. The 3-cell $C$ of $P_{\sigma}$ which contains $v$ is a quotient of an alternating suspension $B$ of a $2 k$-gon with cone points mapping to $u$ and $v$, the identifications arising as follows. If $f_{i}=f_{j}$ for some $i, j \in\{1, \ldots, k\}$, then $u_{i}=u_{j}$, and so the edge joining $u$ and $u_{i}$ equals the edge joining $u$ and $u_{j}$. If $v_{i}=v_{j}$ for some $i, j \in\{1, \ldots, k\}$, then the face containing $u$ and $v_{i}$ equals the face containing $u$ and $v_{j}$. So the 3 -cell of $P_{\sigma}$ which contains $v$ is a quotient of an alternating suspension of a $2 k$-gon with cone points mapping to $u$ and $v$. The quotient map performs two kinds of identifications. Edges containing the cone point which maps to $u$ are identified if some face of $P$ is not locally an embedded disk at $v$. Faces containing the cone point which maps to $u$ are identified if some edge of $P$ is not locally an embedded line segment at $v$. In every case the restriction of the quotient map to every open cell of the alternating suspension is a homeomorphism. Since the identifications are along edges and faces containing $u$, the map $\theta: \operatorname{star}\left(u^{\prime}, B_{\sigma}\right) \rightarrow B$ can be defined so that it induces a cellular homeomorphism $\psi_{C}: \operatorname{star}\left(u, C_{\sigma}\right) \rightarrow C$. 


\subsection{Central balls}

In this subsection and the next we investigate the second dual cap subdivision of a faceted 3-ball.

Let $P$ be a faceted 3-ball. Let $u$ be the vertex of $P_{\sigma}$ which is the barycenter of the 3-cell of $P$. Let $C$ be a 3-cell of $P_{\sigma}$. Section 3.2 shows that $C$ contains $u, C$ is a quotient of an alternating suspension $B$, and there is a cellular homeomorphism $\psi_{C}$ : $\operatorname{star}\left(u, C_{\sigma}\right) \rightarrow C$ which keeps vertices in their cells. These homeomorphisms can be defined compatibly on the pairwise intersections of their domains so that they piece together to give a cellular homeomorphism $\psi: \operatorname{star}\left(u, P_{\sigma^{2}}\right) \rightarrow P_{\sigma}$ which keeps vertices in their cells. We call $\operatorname{star}\left(u, P_{\sigma^{2}}\right)$ the central ball of $P_{\sigma^{2}}$. We have just shown that the central ball of $P_{\sigma^{2}}$ is cellularly homeomorphic to $P_{\sigma}$ in a way which is canonical on vertices.

\subsection{Chimneys}

Let $P$ be a faceted 3 -ball. Let $u$ be the vertex of $P_{\sigma}$ which is the barycenter of the 3-cell of $P$. Let $A_{1}$ be the star of $u$ in the 1 -skeleton of $P_{\sigma}$. Let $A=\operatorname{star}\left(A_{1}, P_{\sigma^{2}}\right)$. We call $A$ the chimney assembly for $P$. This subsection is devoted to investigating the structure of chimney assemblies.

Let $f$ be a face of $P$, and let $a$ be the vertex of $P_{\sigma}$ which is the barycenter of $f$. Then $\operatorname{star}\left(a, P_{\sigma^{2}}\right)$ is a subcomplex of $A$, which we call the $f$-chimney of $A$.

Let $f$ be a face of $P$. Let $F$ be a CW complex such that $F$ is a closed disk, the interior of $F$ is the unique open 2-cell of $F$, and there exists a continuous cellular map $\varphi: F \rightarrow f$ such that the restriction of $\varphi$ to every open cell of $F$ is a homeomorphism. Given a dual cap subdivision $f_{\sigma}$ of $f$, we choose a dual cap subdivision $F_{\sigma}$ of $F$ so that $\varphi$ induces a cellular map $\varphi_{\sigma}: F_{\sigma} \rightarrow f_{\sigma}$. Let $C_{f}$ be the mapping cylinder of $\varphi_{\sigma}$, viewed as a CW complex in the obvious way.

In this and the next four paragraphs we show that $C_{f}$ is cellularly homeomorphic to the $f$-chimney of $A$. Let $a$ be the barycenter of $f$ and let $v$ be a vertex of $f$. Recall from Figure 18 and the discussion in Section 3.1 that there are three possibilities for a face of $\partial P_{\sigma}$. For each of the three possibilities, Figure 22 shows part of $P_{\sigma^{2}}$. Every vertex and edge in Figure 22 is a vertex or edge of $P_{\sigma^{2}}$ except for the dotted arc in the second diagram which joins $b, b^{\prime}$, and $u$. The barycenter $a$ of $f$ is shown. In the first two diagrams $b$ is the barycenter of the edge of $f$ that contains $v$, and $a, b$, and $v$ are the vertices of a face $h$ of $f_{\sigma}$. In the third diagram $b_{1}$ and $b_{2}$ are the barycenters of the two edges of $f$ 

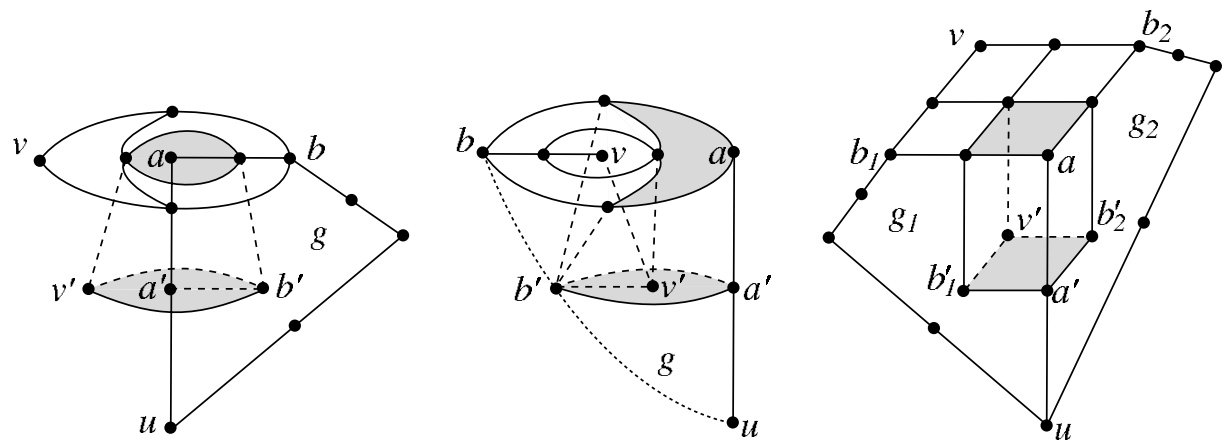

Figure 22: Part of $P_{\sigma^{2}}$

that contain $v$, and $a, b_{1}, b_{2}$, and $v$ are the vertices of a face $h$ of $f_{\sigma}$. The dual cap subdivision of $h$ is shown in Figure 22. The barycenter $u$ of $P$ and $a$ are joined by an edge $e$ of $P_{\sigma}$. Let $a^{\prime}$ be the barycenter of $e$ in $P_{\sigma^{2}}$. Let the map $\psi: \operatorname{star}\left(u, P_{\sigma^{2}}\right) \rightarrow P_{\sigma}$ be as in Section 3.3. Section 3.3 shows that $\psi\left(a^{\prime}\right)=a$. Let $C$ be the 3-cell of $P_{\sigma}$ which contains $v$, and let $v^{\prime}$ be the barycenter of $C$ in $P_{\sigma^{2}}$. Section 3.3 shows that $\psi\left(v^{\prime}\right)=v$. Let $k$ be the face of $h_{\sigma}$ which contains $a$. In each of the three diagrams in Figure 22 we have drawn in gray the face $k$ and a face $h^{\prime}$ which will be described below. We consider separately the three possibilities for $h$ shown in Figure 18.

We first consider the case that $h$ has the form of the first diagram in Figure 18. Then $f$ is a monogon. Let $g$ be the face of $P_{\sigma}$ which contains $a, b$, and $u$, and let $b^{\prime}$ be the barycenter of $g$. For clarity, two edges of $g_{\sigma}$ are not shown. Section 3.3 shows that $\psi\left(b^{\prime}\right)=b$. Let $h^{\prime}$ be the face of $P_{\sigma^{2}}$ with vertices $a^{\prime}, b^{\prime}$, and $v^{\prime}$. Then $k$ and $h^{\prime}$ are cellularly homeomorphic, $\operatorname{star}\left(a, P_{\sigma^{2}}\right)$ is the product of a 1-simplex and the dual cap subdivision of a monogon, and $\operatorname{star}\left(a, P_{\sigma^{2}}\right)$ is cellularly homeomorphic to $C_{f}$.

Now suppose that $h$ has the form of the second diagram in Figure 18. Then $v$ has valence 1 in $\partial f$. As in the previous case let $g$ be the face of $P_{\sigma}$ which contains $a, b$, and $u$, and let $b^{\prime}$ be the barycenter of $g$. Section 3.3 again shows that $\psi\left(b^{\prime}\right)=b$. Let $h^{\prime}$ be the face of $P_{\sigma^{2}}$ with vertices $a^{\prime}, b^{\prime}$, and $v^{\prime}$. Then $k$ is cellularly homeomorphic to a square and $h^{\prime}$ is cellularly homeomorphic to a square with two adjacent edges identified. It follows that the 3-cell of $\operatorname{star}\left(a, P_{\sigma^{2}}\right)$ which contains $v^{\prime}$ is cellularly homeomorphic to a cube with two adjacent edges identified.

Finally, suppose $h$ has the form of the third diagram in Figure 18. For $i \in$ $\{1,2\}$, let $g_{i}$ be the face of $P_{\sigma}$ which contains $u$ and $b_{i}$, and let $b_{i}^{\prime}$ be the 
vertex of $P_{\sigma^{2}}$ which is the barycenter of $g_{i}$. For clarity two edges of $\left(g_{1}\right)_{\sigma}$ and two edges of $\left(g_{2}\right)_{\sigma}$ are omitted in the third diagram in Figure 22. Section 3.3 shows that $\psi\left(b_{i}^{\prime}\right)=b_{i}$ for $i \in\{1,2\}$. Let $h^{\prime}$ be the face of $P_{\sigma^{2}}$ with vertices $a^{\prime}$, $b_{1}^{\prime}, b_{2}^{\prime}$ and $v^{\prime}$. We see that $\psi$ restricts to a cellular homeomorphism from $h^{\prime}$ to $h$. Then both $k$ and $h^{\prime}$ are cellularly homeomorphic to squares and the 3-cell of $\operatorname{star}\left(a, P_{\sigma^{2}}\right)$ which contains $v^{\prime}$ is cellularly homeomorphic to a cube.

If $h$ has the form of the second or third diagram in Figure 18, then $\operatorname{star}\left(a, P_{\sigma^{2}}\right)$ is a union of complexes as described in the previous two paragraphs. It follows in these cases that $\operatorname{star}\left(a, \partial P_{\sigma^{2}}\right)$ is cellularly homeomorphic to $F_{\sigma}$, that the restriction of $\psi$ to $\operatorname{star}\left(a, P_{\sigma^{2}}\right) \cap \operatorname{star}\left(u, P_{\sigma^{2}}\right)$ is a cellular homeomorphism onto $f_{\sigma}$, and that $\operatorname{star}\left(a, P_{\sigma^{2}}\right)$ is cellularly homeomorphic to $C_{f}$.

So the chimney assembly $A$ for $P$ is the union of the central ball of $P_{\sigma^{2}}$ and the chimneys of the faces of $P$. The central ball of $P_{\sigma^{2}}$ is cellularly homeomorphic to $P_{\sigma}$, and the chimneys of the faces of $P$ are mapping cylinders. Figure 23 shows the chimney assembly for a cube.

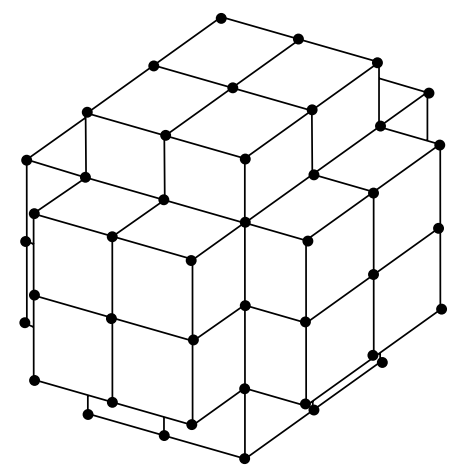

Figure 23: The chimney assembly for a cube

Let $f$ be a face of $P$, and let $C_{f}$ be the $f$-chimney of $A$. We call $f \cap C_{f}$ the top of $C_{f}$. We call the intersection of $C_{f}$ with the central ball of $A$ the bottom of $C_{f}$. We call faces of $\partial C_{f}$ which are in neither the top nor the bottom of $C_{f}$ lateral faces.

\section{Building Heegaard diagrams from face-pairings}

In this section we construct Heegaard diagrams from face-pairings. 


\subsection{Edge pairing surfaces}

We begin by constructing a cellulated closed surface $S$ from a face-pairing. We call $S$ the edge pairing surface of the face-pairing. See the introduction, where $S$ is defined for regular faceted 3-balls. Our more general faceted 3-balls present some complications, but we proceed in much the same way.

Let $P$ be a faceted 3-ball with orientation-reversing face-pairing $\epsilon$. We first construct a cell complex $X$ cellularly homeomorphic to the 1-skeleton of $P$. Let $f$ and $f^{-1}$ be two paired faces of $P$. Next construct a CW complex $F$ such that $F$ is a closed disk, the interior of $F$ is the unique open 2-cell of $F$, and there exists a continuous cellular map $\varphi: F \rightarrow f$ such that the restriction of $\varphi$ to every open cell of $F$ is a homeomorphism. There also exists a corresponding cellular map $\psi: F \rightarrow f^{-1}$ such that $\varphi$ and $\psi$ are related as follows. Recall that to define $\epsilon$ we construct subdivisions $f_{s}$ and $f_{s}^{-1}$ of $f$ and $f^{-1}$ in Section 2. Let $t$ be a face of $f_{s}$. Then there exists a corresponding face $t^{-1}$ of $f_{s}^{-1}$ and a partial face-pairing map $\epsilon_{t}: t \rightarrow t^{-1}$. There also exists a subspace $T$ of $F$ such that the restriction of $\varphi$ to $T$ is a homeomorphism onto $t$. We may, and do, choose the maps $\varphi$ and $\psi$ so that if $x \in T$, then $\psi(x)=\epsilon_{t}(\varphi(x))$. We next construct $\partial F \times[0,1]$. We view the interval $[0,1]$ as a 1-cell, and we view $\partial F \times[0,1]$ as a 2-complex with the product cell structure. For every $x \in \partial F$ we identify $(x, 0) \in \partial F \times[0,1]$ with the point of $X$ corresponding to $\varphi(x) \in \partial f$ and we identify $(x, 1) \in \partial F \times[0,1]$ with the point of $X$ corresponding to $\psi(x) \in \partial f^{-1}$. Doing this for every pair of faces of $P$ yields a cell complex $Y$ whose underlying space is a closed surface. We define $S$ to be the dual cap subdivision of $Y$. We say that an edge of $S$ is vertical if it is either contained in $X$ or is disjoint from $X$. We say that an edge of $S$ is diagonal if it is not vertical. We say that an edge of $S$ is a meridian edge if it is not an edge of $Y$. We refer to edges of $Y$ as nonmeridian edges of $S$. The union of the vertical meridian edges is a family $\left\{\alpha_{1}, \ldots, \alpha_{n}\right\}$ of pairwise disjoint simple closed curves in $S$ called the vertical meridian curves of $S$. The union of the diagonal meridian edges is a family $\left\{\beta_{1}, \ldots, \beta_{m}\right\}$ of pairwise disjoint simple closed curves in $S$ called the diagonal meridian curves of $S$.

Example 4.1.1 We illustrate the above edge pairing surface construction using the simple example of the lens space $L(3,1)$. To obtain $L(3,1)$ we take a faceted 3-ball $P$ with just two faces which are triangles as in Figure 24, where one face is at infinity. The orientation-reversing face-pairing map $\epsilon_{1}$ maps the inner triangle to the outer triangle taking vertex $A$ to vertex $B$. We set $\epsilon=\left\{\epsilon_{1}^{ \pm 1}\right\}$. Let $S$ be the edge pairing surface of $\epsilon$, and let $\widetilde{A}, \widetilde{B}$, and $\widetilde{C}$ be the 


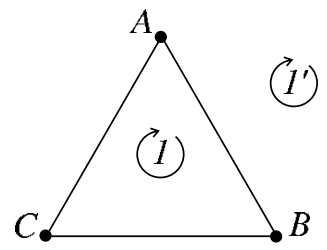

Figure 24: The complex $P$ for Example 4.1.1

vertices of $S$ which correspond to $A, B$, and $C$. Figure 25 shows $S$ as an annulus whose boundary components are to be identified in a straightforward way. Similarly, Figure 26 shows $S$ as a quotient of a quadrilateral. This quadrilateral is gotten from the edge cycle of $\epsilon$, shown in Figure 27, in a straightforward way. The meridian edges of $S$ are drawn with thin arcs, and the nonmeridian edges of $S$ are drawn with thick arcs. We see that $S$ is a torus. The union of the vertical meridian edges is a simple closed curve on the torus, and the union of the diagonal meridian edges is a simple closed curve on the torus. The torus and these two curves form a Heegaard diagram for $L(3,1)$. This is a special case of Theorem 4.2.1.

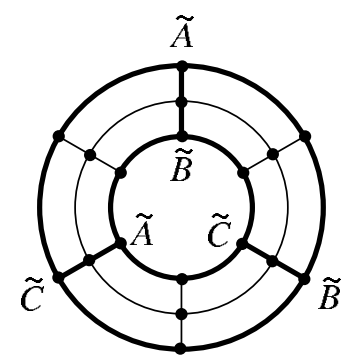

Figure 25: The edge pairing surface of $\epsilon$ viewed as a quotient of an annulus

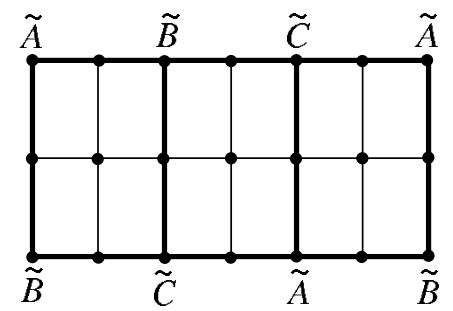

Figure 26: The edge pairing surface of $\epsilon$ viewed as a quotient of a quadrilateral 


$$
\left.\left.\prod_{B}^{A} \stackrel{\varepsilon_{1}}{\rightarrow}\right|_{C} ^{B} \stackrel{\varepsilon_{l}}{\rightarrow} \stackrel{\varepsilon_{l}}{\rightarrow}\right|_{A} ^{A}
$$

Figure 27: A diagram of the edge cycle of $\epsilon$

\subsection{Heegaard diagrams for general face-pairings}

Theorem 4.2.1 Let $P$ be a faceted 3-ball with orientation-reversing facepairing $\epsilon$ such that the cell complex $N=P / \epsilon$ is a manifold with one vertex. Let $H_{1}$ be the star of the barycenter of the 3-cell of $N$ in the 1-skeleton of $N_{\sigma}$, and let $H=\operatorname{star}\left(H_{1}, N_{\sigma^{2}}\right)$. Then

i) $H$ is a handlebody in $N$ and $\partial H$ is a Heegaard surface for $N$, and

ii) $\partial H$ is cellularly homeomorphic to the edge pairing surface $S$ of $\epsilon$.

Identifying $S$ with $\partial H$, we have the following:

iii) the set $\left\{\alpha_{i}\right\}$ of vertical meridian curves of $S$ is a basis of meridian curves for $H$;

iv) the set $\left\{\beta_{i}\right\}$ of diagonal meridian curves of $S$ is a basis of meridian curves for $N \backslash \operatorname{int}(H)$;

v) $\left(S ;\left\{\alpha_{i}\right\} ;\left\{\beta_{i}\right\}\right)$ is a Heegaard diagram for $N$.

Proof We view $N_{\sigma^{2}}$ as a quotient of $P_{\sigma^{2}}$. The preimage of $H$ in $P_{\sigma^{2}}$ is the chimney assembly $A$ for $P$, and $H$ is obtained from the chimney assembly by gluing together the tops in pairs. Hence $H$ is a handlebody in $N$. Let $H^{\prime}$ denote the closure of the complement of $H$ in $N$. Then $H^{\prime}$ is the star in $N_{\sigma^{2}}$ of the 1-skeleton of $N$, and so is a union of stars of vertices and stars of edge barycenters. Each vertex star is a cone with cone point the vertex, and hence is homeomorphic to a closed ball since $N$ is a manifold. Each 3-cell in the star of an edge barycenter is either a cube or (as in the second diagram in Figure 22) a cube with a pair of adjacent edges identified, and the star of each barycenter is a two-sided mapping cylinder obtained from $D^{2} \times I$. It follows that $H^{\prime}$ is a handlebody and so $\partial H$ is a Heegaard surface for $N$. This proves i).

In this paragraph we show that $\partial H$ is cellularly homeomorphic to $S$. The preimage of $\partial H$ in $P_{\sigma^{2}}$ is the union of all the lateral faces of the chimneys of $A$. Section 3.4 shows that every chimney of $A$ is a mapping cylinder, and so the 
union of the lateral faces of every chimney of $A$ is a mapping cylinder. Hence $\partial H$ is homeomorphic to a topological space obtained by attaching two-sided mapping cylinders to the 1-skeleton of $P$. It now follows from the definition of $S$ in terms of attaching two-sided mapping cylinders that $S$ is cellularly homeomorphic to $\partial H$. This proves ii).

Let $f$ be a face of $P$. The top of the $f$-chimney $C_{f}$ of $A$ meets the union of the lateral faces of $C_{f}$ in a simple closed edge path in $A$. This edge path maps to a meridian curve for $H$, and every edge in this meridian curve corresponds to a vertical meridian edge of $S$. Hence the union of the edges of $\partial H$ corresponding to the vertical meridian edges of $S$ forms a basis of meridian curves for $H$. This proves iii).

Suppose given an edge cycle of $\epsilon$ consisting of $j$ distinct edges $e_{1}, \ldots, e_{j}$ of $P$ with diagram

$$
e_{1} \stackrel{\epsilon_{f_{1}}}{\longrightarrow} e_{2} \stackrel{\epsilon_{f_{2}}}{\longrightarrow} \cdots \stackrel{\epsilon_{f_{j-1}}}{\longrightarrow} e_{j} \stackrel{\epsilon_{f_{j}}}{\longrightarrow} e_{1} .
$$

Let $u$ be the vertex of $P_{\sigma^{2}}$ which is the barycenter of the 3-cell of $P$, and let $\psi: \operatorname{star}\left(u, P_{\sigma^{2}}\right) \rightarrow P_{\sigma}$ be the cellular homeomorphism of Section 3.3. Let $e_{i}^{\prime}=\psi^{-1}\left(\left(e_{i}\right)_{\sigma}\right)$, let $v_{i}$ be the vertex of $e_{i}^{\prime}$ such that $\psi\left(v_{i}\right)$ is the barycenter of $e_{i}$ and let $C_{f_{i}}$ be the $f_{i}$-chimney of $A$ for every $i \in\{1, \ldots, j\}$. For every $i \in\{1, \ldots, j\}$ the chimney $C_{f_{i}}$ contains two lateral faces and the chimney $C_{f_{i}^{-1}}$ contains two lateral faces with the following properties, where $i+1$ is taken modulo $j$. See Figure 28. The two lateral faces of $C_{f_{i}}$ both contain an edge which contains $v_{i}$ and a vertex $x_{i}$ in the top of $C_{f_{i}}$, and the two lateral faces of $C_{f_{i}^{-1}}$ both contain an edge which contains $v_{i+1}$ and a vertex $y_{i}$ in the top of $C_{f_{i}^{-1}}$. Furthermore the image in $\partial H$ of $x_{i}$ equals the image in $\partial H$ of $y_{i}$, and both the edge containing $v_{i}$ and $x_{i}$ and the edge containing $v_{i+1}$ and $y_{i}$ map to edges of $\partial H$ which correspond to diagonal meridian edges of $S$. For each $i \in\{1, \ldots, j\}, y_{i-1}, v_{i}, x_{i}$, and the barycenter $b_{i}$ of $e_{i}$ are vertices of face of a $P_{\sigma^{2}}$, where $i-1$ is taken modulo $j$. The union in $N_{\sigma^{2}}$ of the images of these $j$ faces is a properly embedded closed disk $D$ in $H^{\prime}$ whose boundary is in $\partial H^{\prime}=\partial H$. See Figure 29, which shows a chimney assembly together with the top of an $f$-chimney drawn with thick arcs and the face of $P_{\sigma^{2}}$ with vertices $y_{i-1}, v_{i}, x_{i}$, and $b_{i}$. If $\epsilon$ has $m$ edge cycles, then we obtain $m$ such disks $D_{1}, \ldots, D_{m}$ in $H^{\prime}$. The disks $D_{1}, \ldots, D_{m}$ are pairwise disjoint, $H^{\prime} \backslash \bigcup_{i=1}^{m} D_{i}$ has one connected component for every vertex of $N$, and each of these connected components contracts to the corresponding vertex. Since $N$ has only one vertex, it follows that $D_{1}, \ldots, D_{m}$ form a basis of meridian disks for $H^{\prime}$, and so the union of the edges of $\partial H$ corresponding to the diagonal 
meridian edges of $S$ forms a basis of meridian curves for $H^{\prime}$. This proves iv), and v) now follows.
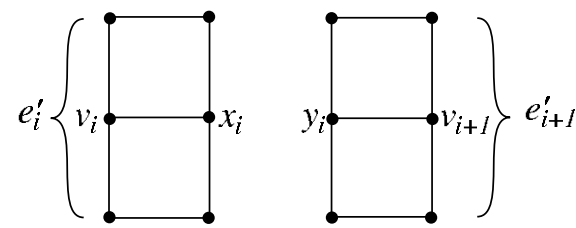

Figure 28: Two lateral faces of $C_{f_{i}}$ and two lateral faces of $C_{f_{i}^{-1}}$

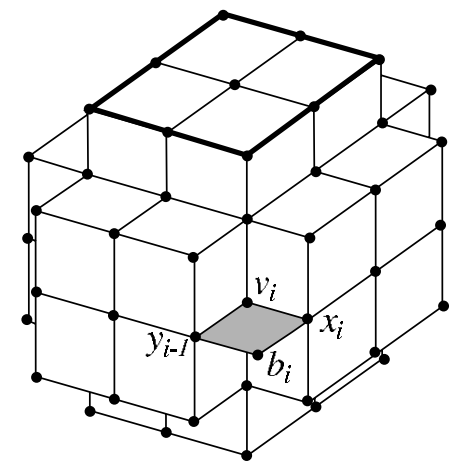

Figure 29: The face with vertices $y_{i-1}, v_{i}, x_{i}$, and $b_{i}$

This proves Theorem 4.2.1.

\subsection{Heegaard diagrams for twisted face-pairing 3-manifolds}

We next interpret Theorem 4.2.1 for twisted face-pairing 3-manifolds.

Let $P$ be a faceted 3-ball with orientation-reversing face-pairing $\epsilon$, and suppose given a multiplier function for $\epsilon$. Let $Q$ be the associated twisted face-pairing subdivision of $P$, let $\delta$ be the associated twisted face-pairing on $Q$, and let $M=Q / \delta$ be the associated twisted face-pairing manifold. Let $S$ be the edge pairing surface of $\delta$.

Theorem 4.2.1 implies that $S$ is cellularly homeomorphic to a Heegaard surface for $M$. We view $S$ as a union of subspaces, one for every edge cycle of $\epsilon$ as follows. Let $E$ be an edge cycle of $\epsilon$. Suppose that $E$ has length $j$, multiplier $k$ and edge cycle diagram

$$
e_{1} \stackrel{\epsilon_{f_{1}}}{\longrightarrow} e_{2} \stackrel{\epsilon_{f_{2}}}{\longrightarrow} \cdots \stackrel{\epsilon_{f_{j-1}}}{\longrightarrow} e_{j} \stackrel{\epsilon_{f_{j}}}{\longrightarrow} e_{1} .
$$


To construct $Q$ from $P$ we subdivide each of the edges $e_{1}, \ldots, e_{j}$ into $j k$ subedges. Every edge of $Q$ gives rise to two edges of $S$. So the edges $e_{1}, \ldots, e_{j}$ of $P$ give rise to subcomplexes $\widetilde{e}_{1}, \ldots, \widetilde{e}_{j}$ of $S$ each of which is the union of $2 j k$ edges of $S$. As in Figure 11 of [2], $\delta$ maps subedge $m$ of $e_{i}$ relative to $f_{i}$ to subedge $m+1$ of $e_{i+1}$ relative to $f_{i+1}$ for every $i \in\{1, \ldots, j\}$ and $m \in\{1, \ldots, j k-1\}$, where $i+1$ is taken modulo $j$. It follows that $E$ gives rise to a subspace $C$ of $S$ as shown in Figure 30. We call $C$ an edge cycle cylinder. Certain arcs contained in $C$ are not edges of $S$, and so they are drawn with dashes. The edges of $S$ are drawn with two thicknesses simply to distinguish the thin meridian edges from the thick nonmeridian edges of $S$. In general $C$ need not be homeomorphic to a closed annulus, but there exists a closed annulus $A$ and a surjective continuous map $\varphi: A \rightarrow C$ such that the restriction of $\varphi$ to the interior of $A$ is a homeomorphism and $\varphi$ maps the boundary of $A$ to the union of the arcs drawn with dashes in Figure 30. We refer to the images under $\varphi$ of the two boundary components of $A$ as the ends of $C$. The ends of $C$ are chosen so that the edge cycle cylinders corresponding to different $\epsilon$-edge cycles meet only along their boundaries and their union is $S$. If $\gamma$ is an $\operatorname{arc}$ in $A$ which joins the boundary components of $A$, then we say that the curve $\varphi(\gamma)$ joins the ends of $C$. If $\gamma$ is a simple closed curve in the interior of $A$ which separates the boundary components of $A$, then we say that the curve $\varphi(\gamma)$ separates the ends of $C$. We define the circumference of $C$ to be $j$, and we define the height of $C$ to be $j k$. Now we see that Figure 11 of [2] essentially shows an edge cycle cylinder in a Heegaard surface for $M$. The thick vertical edges in Figure 30 arise from $P$, and the thick diagonal edges in Figure 30 arise from $P^{*}$. Vertical edges of $S$ are drawn vertically, and diagonal edges of $S$ are drawn diagonally. The following theorem is now clear.

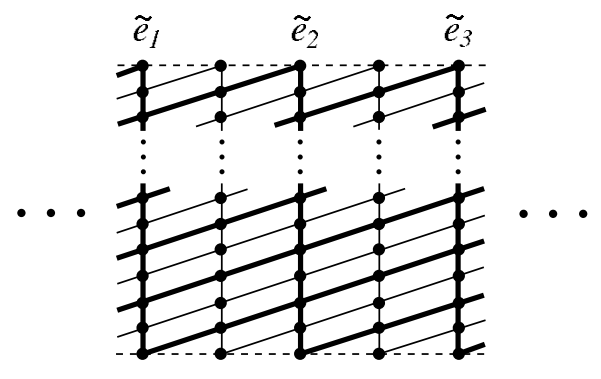

Figure 30: The edge cycle cylinder corresponding to the $\epsilon$-edge cycle $E$

Theorem 4.3.1 Let $M=M(\epsilon, \mathrm{mul})$ be a twisted face-pairing manifold, and let $\delta$ be the associated twisted face-pairing. Let $S$ be the edge pairing surface 
of $\delta$, let $\left\{\alpha_{i}\right\}$ be the set of vertical meridian curves, and let $\left\{\beta_{i}\right\}$ be the set of diagonal meridian curves. Then $\left(S,\left\{\alpha_{i}\right\},\left\{\beta_{i}\right\}\right)$ is a Heegaard diagram for $M$.

Example 4.3.2 We return to Example 2.1. The model face-pairing in Example 2.1 has three edge cycles. Line 2.2 gives diagrams for them. As in Example 2.1, we choose multipliers to be 4, 1, and 1. Each of these three edge cycles gives rise to an edge cycle cylinder as in Figure 30. These three edge cycle cylinders are shown in Figure 31. They are drawn as quadrilaterals with their left sides to be identified with their right sides. The first edge cycle cylinder has circumference 1 and height 4, the second has circumference 2 and height 2 , and the third has circumference 2 and height 2. The thin dotted arcs in Figure 31 indicate how the ends of the cylinders are to be identified. These identifications respect the face-pairing maps, which are also shown. After performing the required identifications we obtain a closed orientable surface $S$ of genus 2 . The union of its vertical meridian edges is a basis of meridian curves for $S$, and the union of its diagonal meridian edges is a basis of meridian curves for $S$. The result is a Heegaard diagram for our twisted face-pairing manifold.
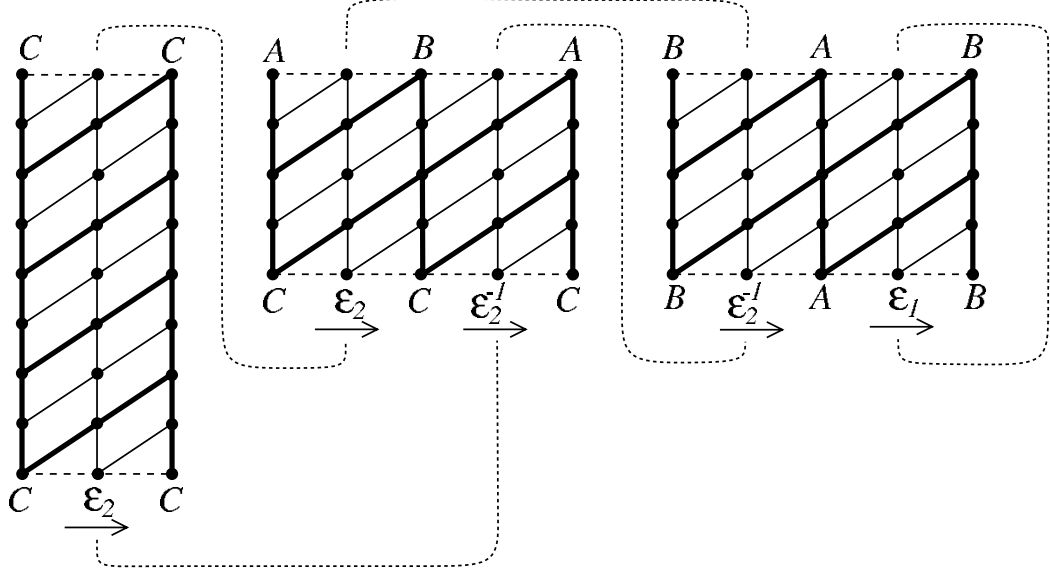

Figure 31: A Heegaard diagram decomposed into three edge cycle cylinders

\section{Building face-pairings from Heegaard diagrams}

In Section 4 we construct Heegaard diagrams from face-pairings. Theorem 4.3.1 shows that every twisted face-pairing manifold has a Heegaard diagram which can be decomposed into cylinders which correspond to the edge cycles of the 
model face-pairing. The height of every such cylinder is a multiple of its circumference, the multiple being the multiplier of the corresponding edge cycle. In this section we show that the decomposition of Heegaard diagrams into analogous cylinders is a general phenomenon, not one restricted to twisted facepairing manifolds. In general the heights of the cylinders need not be multiples of their circumferences. In fact, Theorem 5.3.1 shows that the height of every such cylinder coming from a given Heegaard diagram is a multiple of its circumference if and only if the Heegaard diagram arises from a twisted face-pairing manifold as in Theorem 4.3.1. This provides a characterization of the Heegaard diagrams which we construct for twisted face-pairing manifolds.

\subsection{Generalities concerning Heegaard diagrams}

For us a Heegaard diagram is a Heegaard diagram for a closed connected orientable 3-manifold. It consists of an orientable connected closed surface $S$ with positive genus and two bases of meridian curves for $S$. We assume that there exists a triangulation of $S$ for which each of these meridian curves is piecewise linear, and we assume that these curves intersect transversely in only finitely many points. Let $U$ be the union of the two bases of meridian curves for $S$. We say that our Heegaard diagram is irreducible if every connected component of $S \backslash U$ is homeomorphic to an open disk.

Suppose given an irreducible Heegaard diagram consisting of an orientable connected closed surface $S$ and two bases of meridian curves for $S$. We refer to the meridian curves in one basis as vertical meridian curves, and we refer to the meridian curves in the other basis as diagonal meridian curves. The assumptions imply that the meridian curves of our Heegaard diagram determine a cell structure on $S$ whose vertices are the intersections of the meridian curves and whose faces are the closures of the connected components of the complement in $S$ of the union of the meridian curves. We refer to the edges of $S$ which are contained in vertical meridian curves as vertical (meridian) edges, and we refer to the edges of $S$ which are contained in diagonal meridian curves as diagonal (meridian) edges. Since the meridian curves intersect transversely, every vertex of $S$ has valence 4 and the edges of every face of $S$ are alternately vertical and diagonal. Since the Heegaard diagram is irreducible, no face can have a single edge and so every face of $S$ has an even number of edges.

Algebraic 83 Geometric Topology, Volume 3 (2003) 


\section{$5.2 \quad$ Heegaard cylinders}

Suppose given an irreducible Heegaard diagram with surface $S$. We view $S$ as having a cell structure as in the last paragraph. This subsection is devoted to defining subspaces of $S$ called Heegaard cylinders.

In this paragraph we construct what we call temporary horizontal segments of $S$. For this we choose an orientation of $S$. This orientation of $S$ determines an orientation of the boundary of every face of $S$. Let $f$ be a face of $S$. Let $v_{1}$ be a vertex of $f$ such that a diagonal edge $e_{1}$ of $f$ follows $v_{1}$ (relative to $f$ ). See Figure 32, where, as usual, faces are oriented in the clockwise direction. The vertex $v_{1}$ and the edge $e_{1}$ determine a vertical edge $e_{2}$ of $f$ which follows $e_{1}$ (relative to $f$ ) and a terminal vertex $v_{2}$ of $e_{2}$ (relative to $f$ ). We choose an open arc in the interior of $f$ whose closure joins $v_{1}$ and $v_{2}$. We call the closure of this open arc a temporary horizontal segment of $S$. In Figure $32, e_{1}$ is drawn with a dashed line segment, $e_{2}$ is drawn with a line segment, the rest of the boundary of $f$ is drawn with a broken arc, and the temporary horizontal segment $s$ joining $v_{1}$ and $v_{2}$ is drawn with a dotted line segment. We choose a temporary horizontal segment for every such choice of $e_{1}$ and $e_{2}$ so that the temporary horizontal segments associated to distinct choices of $e_{1}$ and $e_{2}$ meet only at vertices of $S$. Figure 33 shows a complete set of temporary horizontal segments for a digon, a quadrilateral, and a hexagon, with conventions as in Figure 32 .

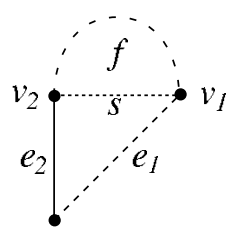

Figure 32: The temporary horizontal segment $s$ of $f$
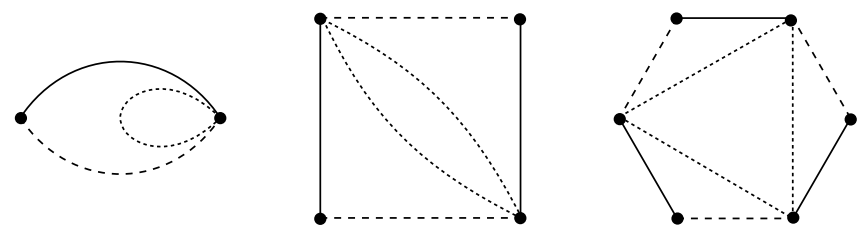

Figure 33: A complete set of temporary horizontal segments for a digon, a quadrilateral and a hexagon

In this paragraph we define what it means for one temporary horizontal segment

Algebraic 83 Geometric Topology, Volume 3 (2003) 
to follow another. Every vertex $v$ of $S$ has a neighborhood as in Figure 34 . The vertex $v$ is contained in temporary horizontal segments $s_{1}, s_{2}, s_{3}$, and $s_{4}$, which need not be distinct. Rotating about $v$ in the clockwise direction from $s_{1}$, we encounter a vertical edge, then a diagonal edge and then $s_{2}$. We say that $s_{2}$ follows $s_{1}$ and likewise that $s_{4}$ follows $s_{3}$. If faces are oriented in the counterclockwise direction, then we rotate about $v$ in the counterclockwise direction. For every temporary horizontal segment $s_{1}$ of $S$ there exists a unique temporary horizontal segment $s_{2}$ of $S$ such that $s_{2}$ follows $s_{1}$. Furthermore, $s_{1}$ is the unique temporary horizontal segment of $S$ such that $s_{2}$ follows $s_{1}$.

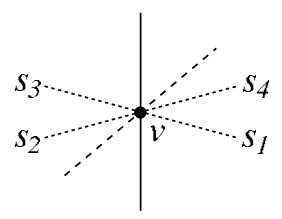

Figure 34: A neighborhood of a vertex $v$ of $S$

In this paragraph we use the temporary horizontal segments of $S$ to construct annuli in $S$. For this let $s_{1}$ be a temporary horizontal segment of $S$. The previous paragraph implies that there exist temporary horizontal segments $s_{2}, \ldots, s_{k}$ such that $s_{i+1}$ follows $s_{i}$ for every $i \in\{1, \ldots, k\}$, where $i+1$ is taken modulo $k$. The union of $s_{1}, \ldots, s_{k}$ is a closed curve $\sigma$ which intersects itself at most tangentially, not transversely. The temporary horizontal segment $s_{1}$ is contained in a face $f$ of $S$, and $s_{1}$ is related to a diagonal edge $e$ of $f$ as in Figure 35. Across $e$ from $f$ is a face $f^{\prime}$ of $S$, and just as $e$ is related to $s_{1}$, the edge $e$ is related to a temporary horizontal segment $s_{1}^{\prime}$ in $f^{\prime}$ as in Figure 35. Just as $s_{1}$ determines the closed curve $\sigma$, the temporary horizontal segment $s_{1}^{\prime}$ determines a closed curve $\sigma^{\prime}$. The curves $\sigma$ and $\sigma^{\prime}$ are the boundary components of an open annulus in $S$ which contains the interior of $e$.

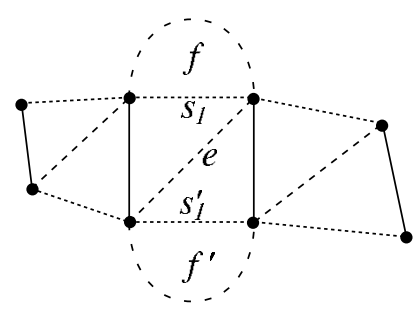

Figure 35: The temporary horizontal segment $s_{1}^{\prime}$ of $f^{\prime}$

A defect of the annuli constructed in the previous paragraph is that the union of their closures is not all of $S$. To remedy this defect, we homotop the temporary 
horizontal segments of $S$ as indicated in Figure 36. More precisely, for every face $f$ of $S$ choose a barycenter $b$ in the open subset of $f$ bounded by temporary horizontal segments and join $b$ with an arc to the initial vertex(s) (relative to $f$ ) of every diagonal edge of $f$ so that these arcs meet only at $b$ and they meet the temporary horizontal segments only at vertices. Then homotop (isotop except for a digon) the temporary horizontal segments of $S$ contained in $f$ to the union of these arcs, fixing endpoints. We refer to the image of a temporary horizontal segment under such a homotopy as a horizontal segment. The result of these homotopies is to enlarge the annuli of the previous paragraph so that the union of their closures is $S$. We refer to the closures of these enlarged annuli as simple cylinders. Each simple cylinder $C$ is the image of a closed annulus $A$ under a continuous map that restricts to a homeomorphism from $\operatorname{int}(A)$ to $\operatorname{int}(C)$. We call the image of each component of $\partial A$ an end of the simple cylinder. Each end of a simple cylinder is a union of horizontal segments.
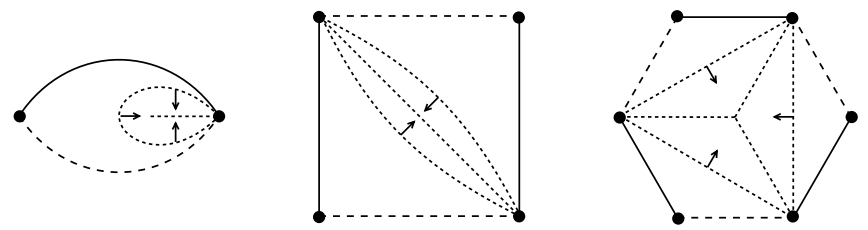

Figure 36: Homotoping the temporary horizontal segments in Figure 33

Suppose that $C_{1}, \ldots, C_{k}$ are simple cylinders, and suppose that $C_{i}$ has ends $E_{i}$ and $E_{i}^{\prime}$ for every $i \in\{1, \ldots, k\}$. Also suppose that the horizontal segments in $E_{i}^{\prime}$ equal the horizontal segments in $E_{i+1}$ for every $i \in\{1, \ldots, k-1\}$. Then we call $C_{1} \cup \cdots \cup C_{k}$ a cylinder. We define a Heegaard cylinder to be a cylinder which is maximal with respect to containment. We define the height of a Heegaard cylinder to be the number of simple cylinders contained in it. We define the circumference of a Heegaard cylinder to be the number of diagonal edges in any simple cylinder contained in the given Heegaard cylinder. The interiors of the simple cylinders of $S$ are pairwise disjoint, and the union of the simple cylinders of $S$ is $S$. It follows that the interiors of the Heegaard cylinders of $S$ are pairwise disjoint, and the union of the Heegaard cylinders of $S$ is $S$.

\subsection{Face-pairings for general Heegaard diagrams}

Theorem 5.3.1 Suppose given an irreducible Heegaard diagram D. Then there exists a faceted 3-ball $P$ with orientation-reversing face-pairing $\epsilon$ such that $N=P / \epsilon$ is a manifold with one vertex and $D$ is the Heegaard diagram of 
$N$ described in Theorem 4.2.1. Furthermore, $D$ is the Heegaard diagram of a twisted face-pairing manifold as described in Theorem 4.3.1 if and only if the height of every Heegaard cylinder of $D$ is a multiple of its circumference.

Proof Let $S$ be the surface of the Heegaard diagram $D$. We begin by defining a 1-complex $K$, which is a subspace of $S$. Recall that homotoping the temporary horizontal segments to the horizontal segments in Section 5.2 involves choosing a barycenter for every face of $S$. These barycenters are the vertices of $K$. The edges of $K$ are dual to the diagonal edges of $S$. In other words, for every diagonal edge $e$ of $S$ there are faces $f_{1}$ and $f_{2}$ of $S$ on either side of $e$, and there is an edge of $K$ corresponding to $e$ which joins the barycenters of $f_{1}$ and $f_{2}$.

Let $V$ be the union of the vertical meridian curves of $D$. Then $S \backslash V$ is homeomorphic to the 2-sphere with $2 g$ holes, where $g$ is the genus of $S$. Of course, we construct $K$ so that $K \subseteq S \backslash V$. Figure 37 indicates how to define a strong deformation retraction from $S \backslash V$ to $K$. In Figure 37 horizontal segments are drawn with dotted arcs, diagonal edges are drawn with dashed arcs, edges of $K$ are drawn with thick arcs, vertical edges are drawn with medium thick arcs and retraction fibers are drawn with thin arcs and dashed arcs. Since filling in the $2 g$ holes of $S \backslash V$ yields a 2-sphere, $K$ is cellularly homeomorphic to the 1-skeleton of a faceted 3-ball $P$ with $2 g$ faces such that a neighborhood of $K$ in $\partial P$ is homeomorphic to $S \backslash V$. We identify $K$ with the 1-skeleton of $P$.
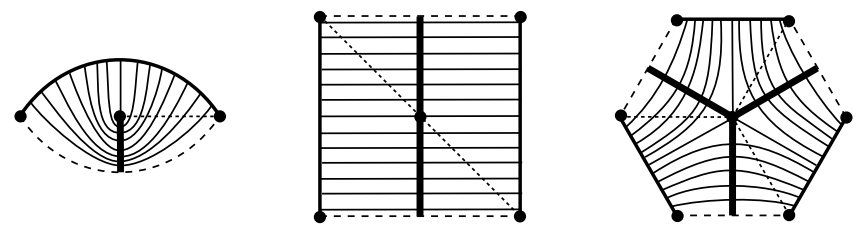

Figure 37: Fibers of a retraction from $S \backslash V$ to $K$

There exists an orientation-reversing face-pairing $\epsilon$ on $P$ which acts on the vertices and edges of $K$ as follows. Let $e$ be an edge of $K$ with vertices $v_{1}$ and $v_{2}$. By definition $e$ is dual to a diagonal edge $d$ of $S$. Let $v$ be a vertex of this diagonal edge of $S$. See Figure 38, where conventions are as in Figure 37. A vertical meridian curve of $D$ passes through $v$. Let $d^{\prime}$ be the diagonal edge of $S$ incident to $v$ across this vertical meridian curve from $d$. Let $e^{\prime}$ be the edge of $K$ dual to $d^{\prime}$, and let $v_{1}^{\prime}$ and $v_{2}^{\prime}$ be the vertices of $e^{\prime}$ corresponding to the vertices $v_{1}$ and $v_{2}$ of $e$ as in Figure 38. The vertex $v$ determines a face $f$ of 
$P$ which contains $e$ and a face $f^{-1}$ which contains $e^{\prime}$. Then the (multivalued) face-pairing map $\epsilon_{f}$ maps $e$ to $e^{\prime}$ taking $v_{1}$ to $v_{1}^{\prime}$ and $v_{2}$ to $v_{2}^{\prime}$.

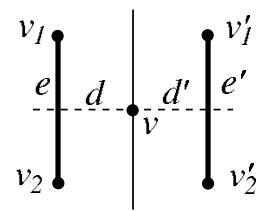

Figure 38: Part of $S$ near $v$

In this paragraph we show that the cell complex $N=P / \epsilon$ is a manifold with one vertex. As in the proof of the main theorem of [1], to prove that $N$ is a manifold, it suffices to prove that the Euler characteristic of $N$ is 0 . It is clear that $N$ has one 3-cell and $g$ faces. So as in the proof of the main theorem of [1], to prove that $N$ is a manifold, it suffices to prove that $N$ has one vertex and $g$ edges. The description of $\epsilon$ in the previous paragraph shows that the $\epsilon$-edge cycles are in bijective correspondence with the diagonal meridian curves of $D$. Since $D$ has $g$ diagonal meridian curves, it follows that $N$ has $g$ edges. Just as we defined the 1-complex $K$ with edges dual to the diagonal edges of $S$, it is possible to define a 1-complex $K^{*}$ with edges dual to the vertical edges of $S$. See Figure 39, which is the same as Figure 38, except that two edges of $K^{*}$ are added as thick dashed line segments. Just as the complex $K$ is connected, so is the complex $K^{*}$. The connectivity of $K^{*}$ and the description of $\epsilon$ in the previous paragraph imply that $N$ has one vertex. Thus $N$ is a manifold with one vertex.

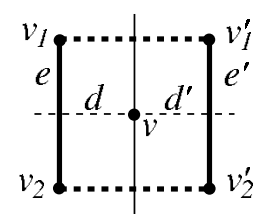

Figure 39: Part of $S$ near $v$

Figure 39 indicates how the edges of $K$ and $K^{*}$ decompose $S$ into a union of quadrilaterals. Furthermore, the vertical and diagonal median curves of $S$ are edges of the dual cap subdivision of this tiling by quadrilaterals. Since the two edges of $K$ that are in a quadrilateral are paired under $\epsilon$ and $K$ is the 1-skeleton of $P$, this exactly matches the cell structure of the edge pairing surface $S^{\prime}$ of $\epsilon$ as the dual cap subdivision of a tiling by quadrilaterals. Hence there exists a homeomorphism from $S$ to $S^{\prime}$ such that the vertical edges of $S$ 
map to vertical meridian edges of $S^{\prime}$ and diagonal edges of $S$ map to diagonal meridian edges of $S^{\prime}$. It follows that $D$ is the Heegaard diagram of $N$ described in Theorem 4.2.1.

Now suppose that $\epsilon$ is a twisted face-pairing. By the previous paragraph, we can identify $S$ with the edge pairing surface $S^{\prime}$ of $\epsilon$. Each edge cycle cylinder is a union of simple cylinders, so every Heegaard cylinder of $D$ is a union of edge cycle cylinders. Since the height of every edge cycle cylinder is a multiple of its circumference, the height of every Heegaard cylinder is a multiple of its circumference.

Finally suppose that the height of every Heegaard cylinder of $D$ is a multiple of its circumference. Let $K^{\prime}$ be the 1-complex with the same underlying space as $K$ but such that each open edge of $K^{\prime}$ is a component of the intersection of $K$ with the interior of a Heegaard cylinder. Let $P^{\prime}$ be the faceted 3-ball with the same underlying space as $P$, but with edges the edges of $K^{\prime}$ rather than the edges of $K$. Before we constructed a face-pairing $\epsilon$ on $P$ by identifying edges of $K$ along diagonal meridian edges. Now we construct a face-pairing $\epsilon^{\prime}$ on $P^{\prime}$ by identifying edges of $K^{\prime}$ along horizontal segments. Then two edges of $K^{\prime}$ are in the same edge cycle exactly if they are in the same Heegaard cylinder, and $\epsilon$ is the twisted face-pairing obtained from $\epsilon^{\prime}$ by choosing the multiplier of an edge cycle to be the quotient of the height of the corresponding Heegaard cylinder by its circumference.

This proves Theorem 5.3.1.

\section{Surgery descriptions for twisted face-pairing man- ifolds}

The Heegaard diagrams of twisted face-pairing manifolds described in Theorem 4.3.1 easily yield surgery descriptions for these manifolds. This section deals with these surgery descriptions.

\subsection{Initial surgery descriptions}

Let $P$ be a faceted 3-ball with orientation-reversing face-pairing $\epsilon$, and suppose given a multiplier function for $\epsilon$. Let $M$ be the associated twisted face-pairing manifold. Theorem 4.3.1 describes a Heegaard diagram $D$ for $M$. Let $S$ be the surface of $D$, let $C$ be an edge cycle cylinder of $D$, and let $\alpha$ be a minimal 
union of vertical meridian edges of $C$ which joins the ends of $C$. Figure 40 shows $C$ as a quadrilateral whose left and right sides are to be identified, and $\alpha$ is shown as a union of vertical dotted edges. Let $\alpha^{\prime}$ be the minimal union of diagonal meridian edges of $C$ which joins the endpoints of $\alpha$ as in Figure 40. Let $\beta$ be a simple closed curve in $C$ which separates the ends of $C$. If the height of $C$ equals the circumference of $C$, then $\alpha^{\prime}$ is isotopic (relative endpoints) to a Dehn twist of $\alpha$ along $\beta$. In this case, let $\tau$ be the appropriate Dehn twist so that $\alpha^{\prime}$ is isotopic (relative endpoints) to $\tau(\alpha)$. In general, if the $\epsilon$-edge cycle corresponding to $C$ has multiplier $m$, then the height of $C$ divided by the circumference of $C$ equals $m$ and $\alpha^{\prime}$ is isotopic (relative endpoints) to $\tau^{m}(\alpha)$ for the appropriate Dehn twist $\tau$ along $\beta$. In Figure 40, $m=2$. This discussion essentially proves the following theorem.

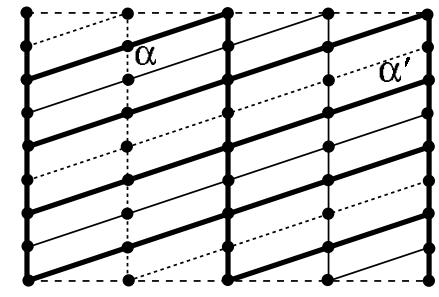

Figure 40: The curves $\alpha$ and $\alpha^{\prime}$

Theorem 6.1.1 Let $M=M(\epsilon, \mathrm{mul})$ be a twisted face-pairing manifold. Let $S$ be the associated Heegaard surface, and let $\alpha_{1}, \ldots, \alpha_{n}$ be the vertical meridian curves. Let $\tau^{\mathrm{mul}}=\tau_{1}^{\mathrm{mul}\left(E_{1}\right)} \circ \cdots \circ \tau_{m}^{\mathrm{mul}\left(E_{m}\right)}$, where $\tau_{1}, \ldots, \tau_{m}$ are consistently oriented Dehn twists along the core curves of the edge cycle cylinders corresponding to the edge cycles $E_{1}, \ldots, E_{m}$. Then $\left(S ;\left\{\alpha_{i}\right\},\left\{\tau^{m u l}\left(\alpha_{i}\right)\right\}\right)$ is a Heegaard diagram for $M$.

Proof The theorem follows from the previous discussion except for the matter of the directions of the Dehn twists. The previous discussion shows that every edge cycle cylinder determines a Dehn twist. Since the twisting directions of these Dehn twists are consistent relative to a fixed orientation of $S$, there are two choices for $\tau^{\mathrm{mul}}$. For one choice of $\tau^{\mathrm{mul}}$ the curves $\tau^{\mathrm{mul}}\left(\alpha_{1}\right), \ldots, \tau^{\mathrm{mul}}\left(\alpha_{n}\right)$ are isotopic to the diagonal meridian curves of $D$, and Theorem 6.1.1 is clear. For the other choice of $\tau^{\mathrm{mul}}$ the curves $\tau^{\mathrm{mul}}\left(\alpha_{1}\right), \ldots, \tau^{\mathrm{mul}}\left(\alpha_{n}\right)$ are isotopic to the diagonal meridian curves, not of $D$, but of the corresponding Heegaard diagram for the twisted face-pairing manifold $M^{*}$ dual to $M$. Theorem 6.1.1 follows because Theorem 4.6 of [2] (together with its generalization in Section 2 if $P$ isn't regular) shows that $M^{*}$ is homeomorphic to $M$. 
We are now prepared for the following theorem, which shows how to obtain twisted face-pairing manifolds by Dehn surgery on framed links in $S^{3}$. We begin with some terminology.

Let $\left(H, H^{\prime} ; \gamma_{1}, \ldots, \gamma_{n} ; \gamma_{1}^{\prime}, \ldots, \gamma_{n}^{\prime}\right)$ be a Heegaard splitting of $S^{3}$. That is, $H$ and $H^{\prime}$ are handlebodies in $S^{3}$ such that $H \cup H^{\prime}=S^{3}$ and $H \cap H^{\prime}=\partial H=\partial H^{\prime}$, $\gamma_{1}, \ldots, \gamma_{n}$ bound a basis of meridian disks for $H$, and $\gamma_{1}^{\prime}, \ldots, \gamma_{n}^{\prime}$ bound a basis of meridian disks for $H^{\prime}$. We call $\left(H, H^{\prime} ; \gamma_{1}, \ldots, \gamma_{n} ; \gamma_{1}^{\prime}, \ldots, \gamma_{n}^{\prime}\right)$ a standard Heegaard splitting of $S^{3}$ if $\gamma_{i}$ and $\gamma_{j}^{\prime}$ are disjoint if $i \neq j$ and $\gamma_{i} \cap \gamma_{j}^{\prime}$ intersect in a single point if $i=j$.

If $H$ is a handlebody and $\beta_{1}, \ldots, \beta_{m}$ are pairwise disjoint simple closed curves in $\partial H$, then pairwise disjoint simple closed curves $\delta_{1}, \ldots, \delta_{m}$ in the interior of $H$ are parallel copies of $\tau_{1}, \ldots, \tau_{m}$ if they satisfy the following: there are pairwise disjoint closed annuli $A_{1}, \ldots, A_{m}$ in $H$ such that $\partial A_{i}=\delta_{i} \cup \tau_{i}$ and $\beta_{i}=A_{i} \cap \partial H$ for every $i \in\{1, \ldots, m\}$.

Theorem 6.1.2 Let $M=M(\epsilon, \mathrm{mul})$ be a twisted face-pairing manifold, let $E_{1}, \ldots, E_{m}$ be the edge cycles of $\epsilon$, let $S$ be the edge pairing surface for the twisted face pairing $\delta$, let $\alpha_{1}, \ldots, \alpha_{n}$ be the vertical meridian curves of $S$, and let $\beta_{1}, \ldots, \beta_{m}$ be core curves for the edge cycle cylinders corresponding to $E_{1}, \ldots, E_{m}$. Let $\left(H, H^{\prime} ; \gamma_{1}, \ldots, \gamma_{n} ; \gamma_{1}^{\prime}, \ldots, \gamma_{n}^{\prime}\right)$ be a standard Heegaard diagram of $S^{3}$. Let $\varphi: S \rightarrow \partial H$ be a homeomorphism such that $\varphi\left(\alpha_{i}\right)=\gamma_{i}$ for every $i \in\{1, \ldots, n\}$, and let $\delta_{1}, \ldots, \delta_{m}$ be parallel copies of $\varphi\left(\beta_{1}\right), \ldots, \varphi\left(\beta_{m}\right)$ in the interior of $H$. We obtain a link $L$ in $S^{3}$ by taking $L=\gamma_{1} \cup \ldots \cup \gamma_{n} \cup \delta_{1} \cup \ldots \cup \delta_{m}$. We define a framing of $L$ as follows. The components $\gamma_{1}, \ldots, \gamma_{n}$ have framing 0 . For every $i \in\{1, \ldots, m\}$ the component $\delta_{i}$ has framing $\operatorname{lk}\left(\delta_{i}, \varphi\left(\beta_{i}\right)\right) \pm$ $\operatorname{mul}\left(E_{i}\right)^{-1}$, where $\operatorname{lk}\left(\delta_{i}, \varphi\left(\beta_{i}\right)\right)$ is the linking number of $\delta_{i}$ and $\varphi\left(\beta_{i}\right)$ after they are compatibly oriented and the sign is either + for every $i \in\{1, \ldots, m\}$ or for every $i \in\{1, \ldots, m\}$. Then the manifold obtained by Dehn surgery on this framed link $L$ is homeomorphic to $M$.

Proof The surface $\partial H$ and the curves $\gamma_{1}, \ldots, \gamma_{n}$ and $\gamma_{1}^{\prime}, \ldots, \gamma_{n}^{\prime}$ form a Heegaard diagram for $S^{3}$. By performing Dehn surgery on $\gamma_{1}, \ldots, \gamma_{n}$, each with framing 0 , we obtain a connected sum of $n$ copies of $S^{2} \times S^{1}$, which has a Heegaard diagram consisting of the surface $\partial H$, the curves $\gamma_{1}, \ldots, \gamma_{n}$ and the curves $\gamma_{1}, \ldots, \gamma_{n}$. (The bases of meridian curves are equal.) For every $i \in\{1, \ldots, m\}$ let $\tau_{i}$ be a Dehn twist on $\partial H$ along $\varphi\left(\beta_{i}\right)$, choosing $\tau_{1}, \ldots, \tau_{m}$ so that the directions in which they twist are consistent relative to a fixed orientation of $\partial H$. Set $\tau^{\mathrm{mul}}=\tau_{1}^{\operatorname{mul}\left(E_{1}\right)} \circ \cdots \circ \tau_{m}^{\operatorname{mul}\left(E_{m}\right)}$. Theorem 6.1.1 implies that $M$ has a Heegaard diagram consisting of the surface $\partial H$, the curves $\gamma_{1}, \ldots, \gamma_{n}$ 
and the curves $\tau^{\mathrm{mul}}\left(\gamma_{1}\right), \ldots, \tau^{\mathrm{mul}}\left(\gamma_{n}\right)$. The fact that $M$ is obtained by Dehn surgery on $\gamma_{1}, \ldots, \gamma_{n}$ and $\delta_{1}, \ldots, \delta_{m}$ now follows from a standard argument which appears, for example, in the proof of the Dehn-Lickorish Theorem on page 84 of [6]. It only remains to determine the framings of $\delta_{1}, \ldots, \delta_{m}$.

We determine the framings of $\delta_{1}, \ldots, \delta_{m}$ in this paragraph. Let $i \in\{1, \ldots, m\}$. Let $T$ be a solid torus regular neighborhood of $\delta_{i}$ such that $\varphi\left(\beta_{i}\right) \subseteq \partial T$. Let $\alpha \subseteq \partial T$ be the boundary of a meridian disk of $T$. The curve $\alpha$ and part of $\varphi\left(\beta_{i}\right)$ are shown in part a) of Figure 41. Using our usual orientation convention for figures as in Figure 40, our Dehn twist takes $\alpha$ to a curve $\gamma$ as shown in part b) of Figure 41. Let $m=\operatorname{mul}\left(E_{i}\right)$. Then $\gamma$ is homologous in $\partial T$ to $\alpha-m \varphi\left(\beta_{i}\right)=\left(1-m \operatorname{lk}\left(\delta_{i}, \varphi\left(\beta_{i}\right)\right)\right) \alpha-m \ell_{i}$, where $\ell_{i}=\varphi\left(\beta_{i}\right)-\operatorname{lk}\left(\delta_{i}, \varphi\left(\beta_{i}\right)\right) \alpha$ is parallel to $\delta_{i}$ and hence is a longitude for $T$. Thus the framing of $\delta_{i}$ is $\operatorname{lk}\left(\delta_{i}, \varphi\left(\beta_{i}\right)\right)-1 / m$. If our Dehn twist is in the opposite direction, then the framing of $\delta_{i}$ is $\operatorname{lk}\left(\delta_{i}, \varphi\left(\beta_{i}\right)\right)+1 / m$.
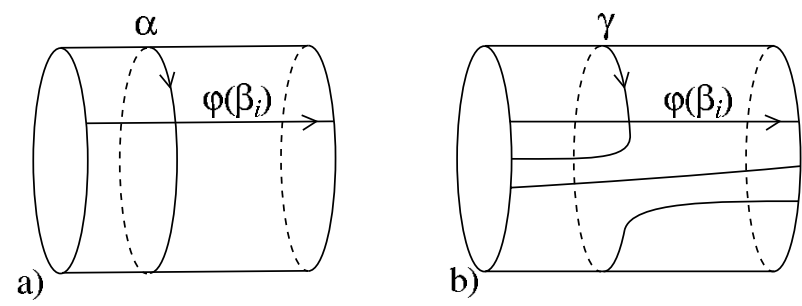

Figure 41: Determining the framing of $\delta_{i}$

This proves Theorem 6.1.2.

\subsection{The corridor construction}

Theorem 6.1.2 describes a framed link $L$ in $S^{3}$ such that Dehn surgery on $L$ obtains a given twisted face-pairing manifold. The goal of this subsection is to make the construction of such links $L$ algorithmic and simple. We call the method which we use the corridor construction.

Let $P$ be a faceted 3-ball, and let $\epsilon$ be an orientation-reversing face-pairing on $P$. In this paragraph we construct corridors between the paired faces of $P$. Let $f$ be a face of $P$. The face $f$ is paired with the face $f^{-1}$. Let $c$ be a corner of $f$ at a vertex $v$ of $f$, and suppose that $\epsilon_{f}$ takes $c$ to the corner $c^{\prime}$ of $f^{-1}$ at the vertex $v^{\prime}$ of $f^{-1}$. Let $\gamma$ be an edge path arc in $P$ with endpoints $v$ and $v^{\prime}$. See the left part of Figure 42, where $f$ and $f^{-1}$ are triangles, $\gamma$ is drawn with thick line segments, and the corners $c$ and $c^{\prime}$ are indicated with dotted 
edges. From $\partial P$ we construct a new cell complex with underlying space the 2 -sphere as follows. We choose an arbitrarily small neighborhood of $\gamma$ in $\partial P$ and modify the cell structure of $\partial P$ only in this neighborhood as indicated in Figure 42. The right part of Figure 42 shows the new cell complex. We refer to this modification of $\partial P$ as constructing a corridor between $f$ and $f^{-1}$. In a straightforward way we continue to successively construct corridors between all the paired faces of $P$. We call the resulting cell complex $C$ a corridor complex for $\epsilon$. Every face of $C$ is in some sense the union of two paired faces of $P$ and a corridor.

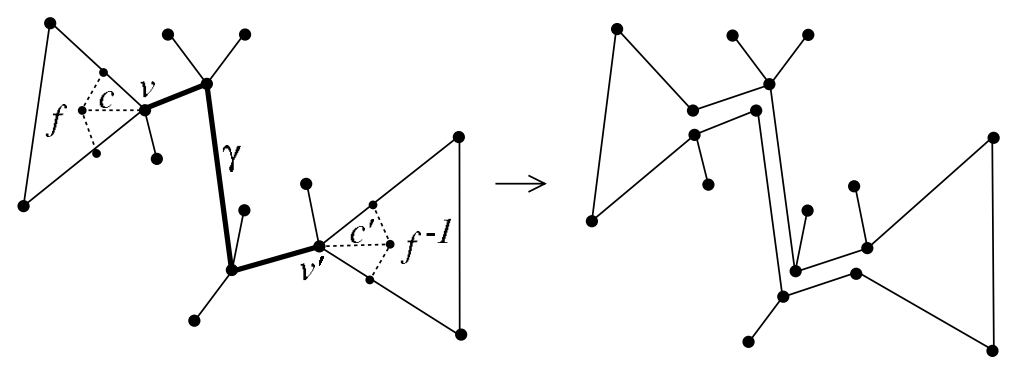

Figure 42: Constructing a corridor between $f$ and $f^{-1}$

Again let $P$ be a faceted 3-ball, and let $\epsilon$ be an orientation-reversing facepairing on $P$. In this paragraph we describe a planar diagram $D$ of a link $L$ in $S^{3}$. Let $C$ be a corridor complex for $\epsilon$. We view the underlying space of $C$ as the one-point compactification $\mathbf{R}^{2} \cup\{\infty\}$ of $\mathbf{R}^{2}$, where the point $\infty$ lies in the interior of some face of $C$. The diagram $D$ lies in $C \backslash\{\infty\}$. Let $g$ be a face of $C$. We next describe the part of $D$ which lies in $g$. One component of $L$ has a projection $\alpha$ in the interior of $g \backslash\{\infty\}$ with no self-crossings; it is unknotted. We call this component of $L$ a face component of $L$. To describe the rest of $D$ which lies in $g$, we construct a continuous map $\varphi: C \rightarrow \partial P$ (which is independent of $g$ ) such that 1) $\varphi$ maps vertices to vertices in the canonical way, 2) the restriction of $\varphi$ to every edge of $C$ is a homeomorphism onto the canonically corresponding edge of $P$ and 3) the restriction of $\varphi$ to the inverse image of the interior of every face of $P$ is a homeomorphism. The face $g$ of $C$ corresponds to two paired faces $f$ and $f^{-1}$ of $P$. Let $c$ be an edge cone of $f$ at an edge $e$ (as defined in the fifth paragraph of Section 2). The face-pairing $\epsilon$ pairs $c$ with an edge cone $c^{\prime}$ of $f^{-1}$ at an edge $e^{\prime}$. Then part of one component of $L$ has a projection $\beta$ in $g \backslash\{\infty\}$ such that 1) only the endpoints of $\beta$ lie in an edge of $g, 2) \varphi(\beta)$ begins at the barycenter of $e, 3)$ then an initial segment of $\varphi(\beta)$ lies in $c, 4)$ then $\beta$ crosses under $\alpha, 5)$ then $\beta$ crosses over $\alpha, 6$ ) then a terminal segment of $\varphi(\beta)$ lies in $c^{\prime}$ and 7) finally $\varphi(\beta)$ ends at the barycenter 
of $e^{\prime}$. The corridor complex $C$ is constructed so that we may, and do, choose the projections $\beta$ for a fixed $g$ (and $f$ ) and varying $c$ to have no self-crossings and no crossings with each other. Figure 43 shows the face component $\alpha$ and the projections $\beta$ for the face on the right part of Figure 42 . Constructing such projections for every face $g$ of $C$ obtains $D$. The components of $L$ other than the face components are in bijective correspondence with the edge cycles of $\epsilon$. We call these components of $L$ edge components. We call $D$ a corridor complex link diagram for $\epsilon$. We call $L$ a corridor complex link for $\epsilon$.

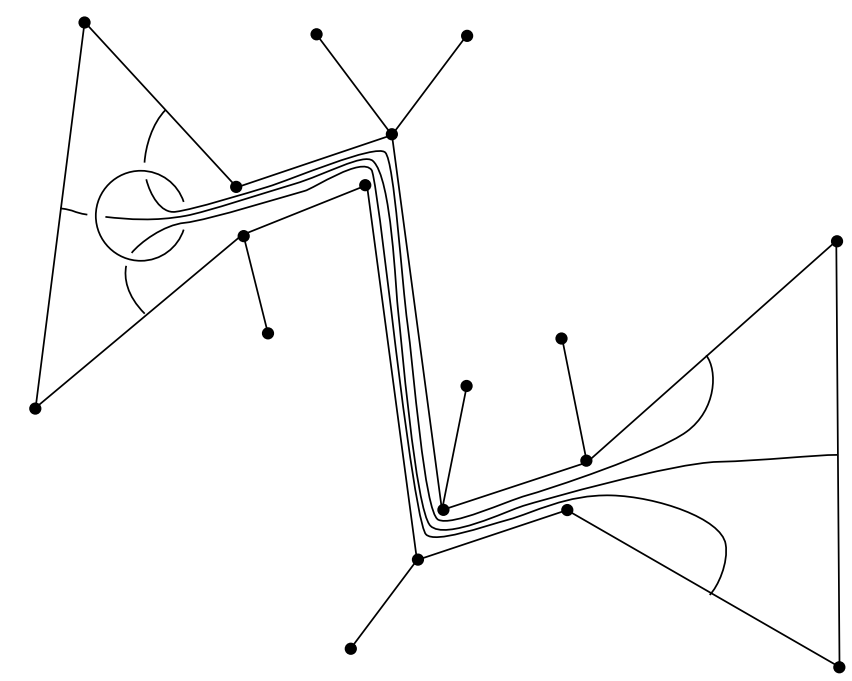

Figure 43: The face component $\alpha$ and the projections $\beta$

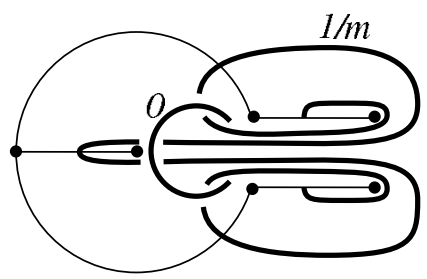

Figure 44: A framed corridor complex link diagram for Example 2.3

Example 6.2.1 We return to the model face-pairing in Example 2.3. A corridor complex for it appears in Figure 44, drawn with thin arcs. A framed corridor complex link diagram for it also appears in Figure 44, drawn with thick arcs. The model face-pairing has only one edge cycle, and we let it have multiplier $m$. Theorem 6.2.2 states that the associated twisted face-pairing 
manifold $M$ is obtained by Dehn surgery on the framed link in Figure 44. The framed link in Figure 44 is isotopic to a link consisting of two unlinked circles, one with framing 0 and one with framing $1 / \mathrm{m}$. As in Proposition 14.4 of [6], Dehn surgery on a circle in $S^{3}$ with framing 0 gives $S^{2} \times S^{1}$, and as in Proposition 14.6 of [6], Dehn surgery on a circle in $S^{3}$ with framing $1 / m$ gives $S^{3}$. Thus $M$ is the connected sum of $S^{2} \times S^{1}$ and $S^{3}$. In other words, $M$ is $S^{2} \times S^{1}$ for every choice of the multiplier $m$.

Theorem 6.2.2 Let $M=M(\epsilon, \mathrm{mul})$ be a twisted face-pairing manifold, and let $E_{1}, \ldots, E_{m}$ be the edge cycles of $\epsilon$. Let $L$ be a corridor complex for $\epsilon$ with diagram $D$. Define a framing on $L$ as follows: every face component of $L$ has framing 0 , and the edge component of $L$ corresponding to $E_{i}$ has framing mul $\left(E_{i}\right)^{-1}$ plus its blackboard framing relative to $D$ for every $i \in$ $\{1, \ldots, m\}$. Then the manifold obtained by Dehn surgery on the framed link $L$ is homeomorphic to $M$.

Proof Let $C$ be the corridor complex for $\epsilon$ from which $D$ is constructed. As in the construction of $D$, we view the underlying space of $C$ as the one-point compactification $\mathbf{R}^{2} \cup\{\infty\}$ of $\mathbf{R}^{2}$, where the point $\infty$ lies in the interior of some face of $C$. We choose standard coordinates $x, y$ and $z$ for $\mathbf{R}^{3}$, and we identify $C \backslash\{\infty\}$ with the $x y$-plane in $\mathbf{R}^{3}$. We choose a closed standard metric ball in $\mathbf{R}^{3}$ centered at the origin so large that it contains every edge of $C$ in its interior. Let $X$ be the solid hemisphere consisting of all points of this ball on and below the $x y$-plane.

In this paragraph we construct a handlebody in $\mathbf{R}^{3}$ by attaching handles to $X$. Let $f$ and $f^{-1}$ be two paired faces of $P$. Let $g$ be the face of $C$ corresponding to $f$ and $f^{-1}$. If $\infty \notin g$, then $g \subseteq \partial X$. If $\infty \in g$, then $g \cap \partial X$ has nonempty interior and is the complement in $g$ of a neighborhood of $\infty$. We attach a standard handle to $g \cap X$. This handle is embedded in $\mathbf{R}^{3}$ so that its vertical projection to the $x y$-plane lies both in $X$ and in the interior of $g$. Figure 45 gives a view from above of $g$ and the handle attached to $g$, where both $f$ and $f^{-1}$ are squares joined by a simple corridor. Figure 46 gives another view of this handle. For every two paired faces of $P$ we attach a handle to $X$ in this way. We denote the result by $H$. It is clear that $H$ is a handlebody and that the closure of the complement of $H$ in $S^{3}$ is also a handlebody.

We next construct simple closed curves in $\partial H$ as follows. First choose a barycenter for every edge of $C$. Again let $f$ and $f^{-1}$ be two paired faces of $P$, and let $g$ be the corresponding face of $C$. Just as in the construction of $D$, construct curves in $\partial H$ which lie in and above $g$; these curves cross the 


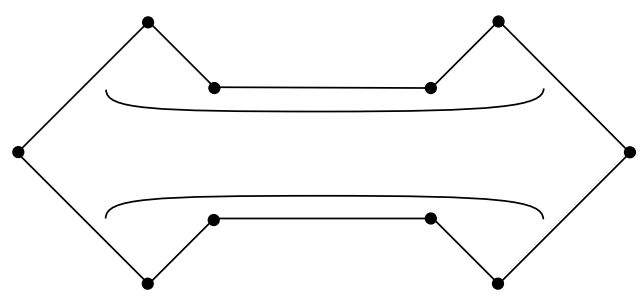

Figure 45: Top view of the handle attached to $g$

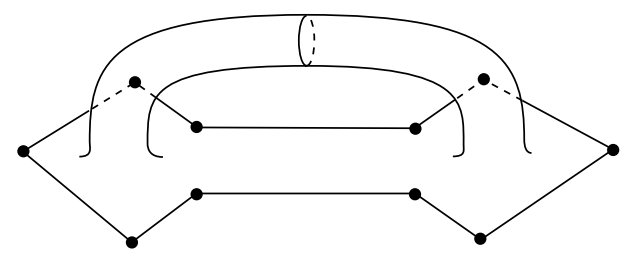

Figure 46: Another view of the handle attached to $g$

handle and they join barycenters of edges of $g$ which correspond to edges of $f$ and barycenters of edges of $g$ which correspond to edges of $f^{-1}$. For every corridor edge $e$ of $g$ construct an arc in $g$ in the obvious way which joins the barycenter of $e$ and the barycenter of the edge of $g$ across the corridor from $e$. We construct all these curves so that only their endpoints lie in edges of $g$ and they are pairwise disjoint except possibly at endpoints. Finally, construct a meridian curve for the handle of $H$ attached to $g$ such that this meridian curve meets each of the curves which cross the handle exactly once. Figure 47 shows a top view of $g$ and the handle of $H$ attached to $g$ with the curves just constructed drawn with thick solid and dashed arcs. Doing this for every two paired faces of $P$, we obtain two families of simple closed curves in $\partial H$. The curves $\gamma_{1}, \ldots, \gamma_{n}$ in one family are the meridian curves of the handles of $H$. The curves $\delta_{1}, \ldots, \delta_{m}$ in the other family correspond canonically to the edge cycles of $\epsilon$.

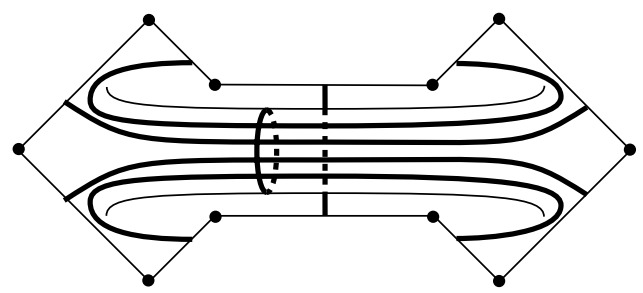

Figure 47: Constructing curves in the part of $\partial H$ which project vertically to $g$ 
Let $S$ be the edge pairing surface of the twisted face-pairing $\delta$, let $\alpha_{1}, \ldots, \alpha_{n}$ be the vertical meridian curves, and let $\beta_{1}, \ldots, \beta_{m}$ be core curves of the edge cycle cylinders. Then $S$ is homeomorphic to the edge pairing surface $S^{\prime}$ of $\epsilon$ by a homeomorphism that takes $\alpha_{1}, \ldots, \alpha_{n}$ to the vertical meridian curves of $S^{\prime}$ and takes $\beta_{1}, \ldots, \beta_{m}$ to the diagonal meridian curves of $S^{\prime}$. The surface $\partial H$ is homeomorphic to the edge pairing surface $S^{\prime}$ of $\epsilon$ by a homeomorphism that takes $\gamma_{1}, \ldots, \gamma_{n}$ to the vertical meridian curves of $S^{\prime}$ and takes $\delta_{1}, \ldots, \delta_{m}$ to the diagonal meridian curves of $S^{\prime}$. Hence the curves $\gamma_{1}, \ldots, \gamma_{n}$ and $\delta_{1}, \ldots, \delta_{m}$ can be indexed so that there exists a homeomorphism $\varphi: S \rightarrow \partial H$ such that $\varphi\left(\alpha_{i}\right)=\gamma_{i}$ and $\varphi\left(\beta_{j}\right)=\delta_{j}$ for every $i \in\{1, \ldots, n\}$ and $j \in\{1, \ldots, m\}$. Theorem 6.1.2 produces a framed link $L$ in $S^{3}$ such that the manifold obtained by Dehn surgery on $L$ is homeomorphic to $M$. Finally, it is clear that $D$ is a diagram of $L$ and that the framings are as claimed.

This proves Theorem 6.2.2.

\section{Examples}

In this section we present some examples in which we use Theorem 6.2.2 to identify some twisted face-pairing manifolds. We have already given such an example in Example 6.2.1, where we constructed a framed link in $S^{3}$ for the model face-pairing in Example 2.3. Using this we showed that the twisted face-pairing manifolds in Example 2.3 are all homeomorphic to $S^{2} \times S^{1}$.

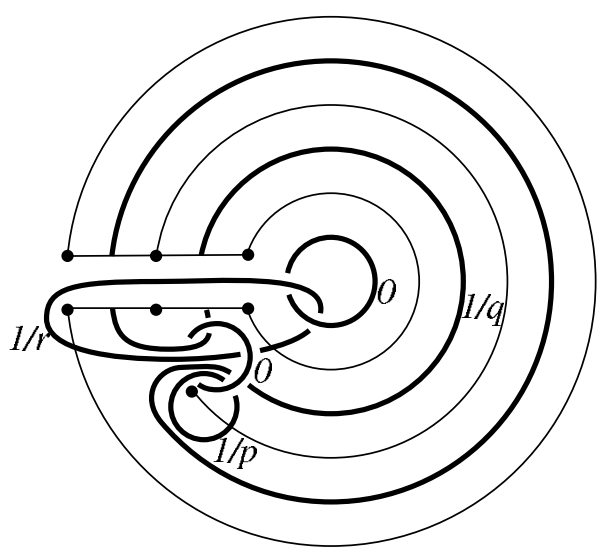

Figure 48: The corridor complex and framed link diagram for Example 2.1 




Figure 49: A simpler framed link

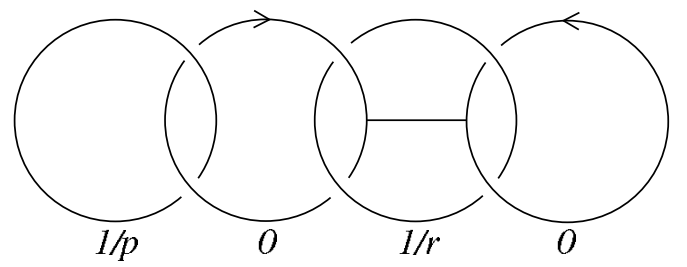

Figure 50: Preparing for a Kirby move of type 2
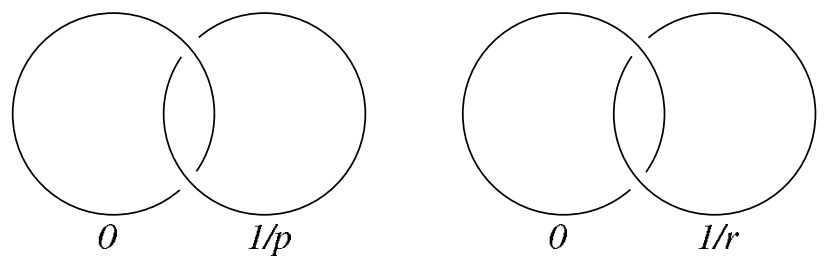

Figure 51: A simpler framed link

Example 7.1 We return to the model face-pairing in Example 2.1. We choose multipliers of the edge cycles in line 2.2 to be $p, q$, and $r$, in order. A corridor complex for Example 2.1 appears in Figure 48, drawn with thin arcs. A framed link diagram for it also appears in Figure 48, drawn with thick arcs. Theorem 6.2.2 states that the associated twisted face-pairing manifold $M$ is obtained by Dehn surgery on the framed link in Figure 48. The framed link in Figure 48 is isotopic to the framed link in Figure 49. The component of the link in Figure 49 with framing $1 / q$ corresponds to a connected summand of $M$. But, as in Example 6.2.1, this connected summand is $S^{3}$. So we delete the component of the link in Figure 49 with framing $1 / q$. We modify the component of the link in Figure 49 with framing 0 which links the components with framings $1 / p$ and $1 / r$ by means of a Kirby move of type 2 . For this we orient the components with framing 0 and connect them with an arc as shown in Figure 50. The result is a link isotopic to the one in Figure 51. It follows from Proposition 17.3 of [6] that $M$ is a connected sum of the lens space $L(p,-1)=L(p, 1)$ and 
the lens space $L(r,-1)=L(r, 1)$.

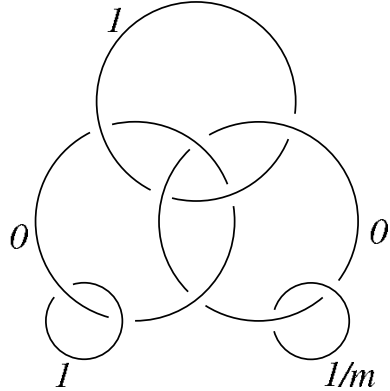

a)

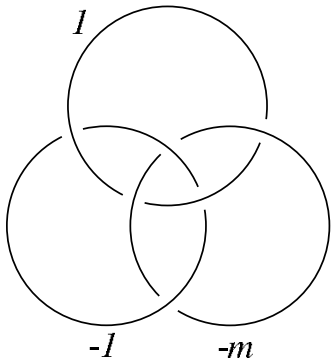

b)

Figure 52: Two framed links for Example 7.2

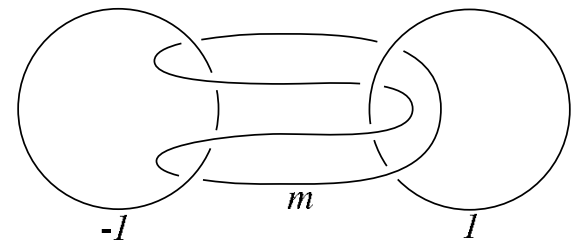

a)

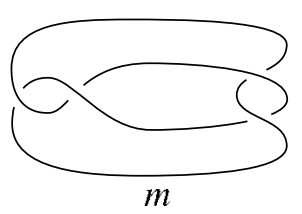

b)

Figure 53: Two more framed links for Example 7.2

Example 7.2 We return to the model face-pairing which we described at the beginning of the introduction. We choose multipliers $m_{1}=1, m_{2}=1$, and $m_{3}=m$. A corridor complex for this example appears in Figure 6, drawn with thin arcs, and a framed link diagram for it also appears in Figure 6, drawn with thick arcs. The part of the link in Figure 6 which is the union of the components with framing 0 and the component which in the diagram crosses both components with framing 0 is isotopic to the Borromean rings. So the framed link in Figure 6 is isotopic to the link in part a) of Figure 52. We simplify the framed link in part a) of Figure 52 using Kirby calculus by performing twist moves, which are discussed in Sections 16.4, 16.5 and 19.4 of [6] under the name Fenn-Rourke moves. Twisting $-m$ times along the component with framing $1 / m$, twisting -1 times along the similar component with framing 1 , and deleting resulting components with framing $\infty$ yields the link in part b) of Figure 52. Because the link in part b) of Figure 52 is amphicheiral we may, and do, multiply every framing by -1 . We isotop the result to the framed link 
in part a) of Figure 53. Now we perform twist moves on the link in part a) of Figure 53. We twist 1 time along the component with framing -1 , twist -1 times along the component with framing 1, and delete resulting components with framing $\infty$. The result is shown in part b) of Figure 53. This is the figure eight knot with framing $m$. If $m=1$, then $M$ is the Brieskorn homology sphere $\Sigma(2,3,7)$, which has the geometry of the universal cover of $\operatorname{PSL}(2, \mathbf{R})$. According to Theorem 4.7 of [7], $M$ is hyperbolic if $m \geq 5$.

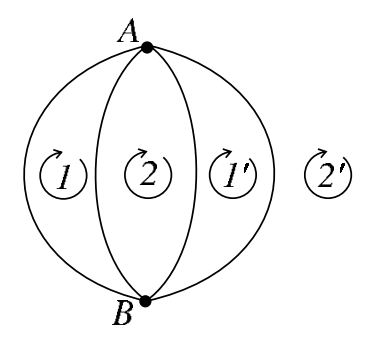

Figure 54: The complex $P$ for Example 7.3

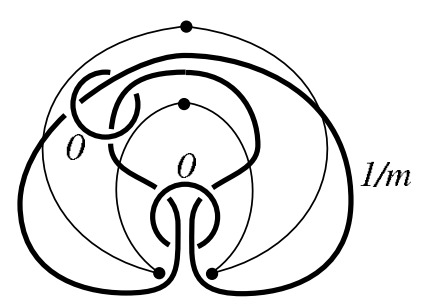

Figure 55: A corridor complex and framed link diagram for Example 7.3

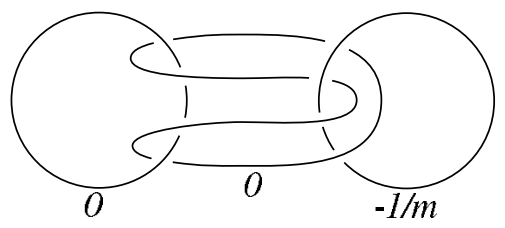

a)

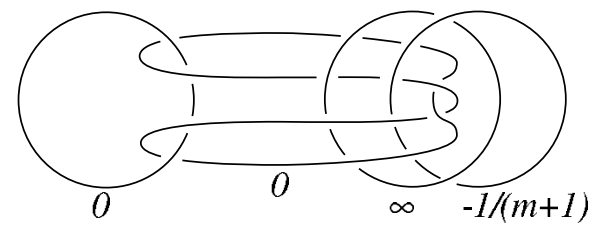

b)

Figure 56: Two framed links for Example 7.3

Example 7.3 This example is closely related to the previous one. The model faceted 3-ball for this example is gotten from the faceted 3-ball given in Figure 1 by collapsing the edge $A B$ to a point and collapsing the edge $C D$ to a point. The result is the faceted 3-ball $P$ given in Figure 54. Because the edges $A B$ 


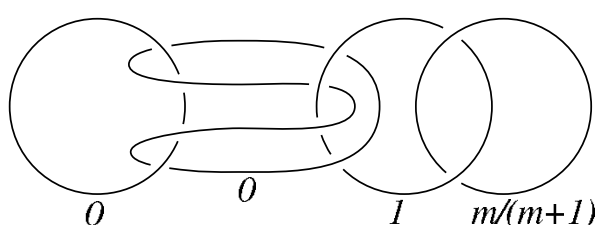

a)

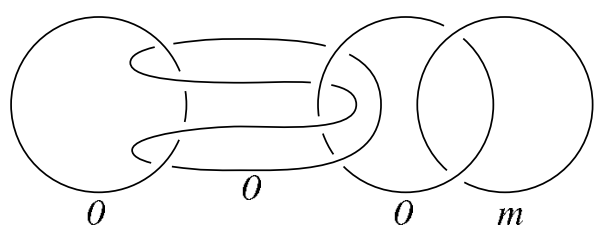

b)

Figure 57: Two more framed links for Example 7.3

and $C D$ in Figure 1 are both fixed by the model face-pairing of Example 7.2, the model face-pairing of Example 7.2 induces a model face-pairing $\epsilon$ on $P$. The face-pairing $\epsilon$ pairs the faces of $P$ as indicated in Figure 54, and the facepairing maps of $\epsilon$ fix the vertices $A$ and $B$. The model face-pairing $\epsilon$ has one edge cycle. This edge cycle has length 4 and corresponds to the edge cycle of length 4 in Example 7.2. We let this edge cycle of $\epsilon$ have multiplier $m$. A corridor complex for $\epsilon$ appears in Figure 55, drawn with thin arcs, and a framed link diagram for it also appears in Figure 55, drawn with thick arcs. This link is the Borromean rings. As in Example 7.2 we may, and do, multiply the framings by -1 and we isotop the link in Figure 55 to obtain the framed link in part a) of Figure 56. Now we perform a twist move by twisting -1 times along the component with framing $-1 / m$ and we introduce a component with framing $\infty$ to obtain the framed link in part b) of Figure 56. Next we twist 1 time along the component with framing $\infty$ to obtain the link in part a) of Figure 57. Finally, we twist -1 times along the component with framing $m /(m+1)$ to obtain the link in part b) of Figure 57. Adding a component with framing $\infty$ that is parallel to the component with framing $m$ gives a special case of the link at the top of Figure 12 on page 146 of [5]. It follows that $M$ is the Seifert fibered manifold $\langle O o 1 \mid 0 ;(m, 1)\rangle$ in the notation of [5]. This means that $M$ is orientable with an orientable base surface of genus 1, that the Euler number of $M$ is 0 , and that $M$ has one exceptional fiber of type $(m, 1)$. When $m=1$, the manifold $M$ is the Heisenberg manifold, the prototype for Nil geometry. 


\section{References}

[1] J W Cannon, W J Floyd, W R Parry, Introduction to twisted face-pairings, Math. Res. Lett. 7 (2000) 477-491

[2] J W Cannon, W J Floyd, W R Parry, Twisted face-pairing 3-manifolds, Trans. Amer. Math. Soc. 354 (2002) 2369-2397

[3] J W Cannon, W J Floyd, W R Parry, Ample twisted face-pairing 3manifolds, preprint

[4] J W Cannon, W J Floyd, W R Parry, A survey of twisted face-pairing 3manifolds, preprint

[5] J M Montesinos, Classical Tesselations and Three-Manifolds, Springer-Verlag, New York (1987)

[6] V V Prasolov, A B Sossinsky, Knots, Links, Braids and 3-Manifolds, Amer. Math. Soc., Providence (1997)

[7] W. P. Thurston, The Geometry and Topology of 3-Manifolds, Princeton lecture notes, (1979) http://www.msri.org/publications/books/gt3m

Department of Mathematics, Brigham Young University

Provo, UT 84602, USA

Department of Mathematics, Virginia Tech

Blacksburg, VA 24061, USA

Department of Mathematics, Eastern Michigan University

Ypsilanti, MI 48197, USA

Email: cannon@math.byu.edu, floyd@math.vt.edu, walter.parry@emich.edu

URL: http://www.math.vt.edu/people/floyd

Received: 12 November $2001 \quad$ Revised: 5 February 2003

Algebraic 6 Geometric Topology, Volume 3 (2003) 